\title{
Parteiendifferenz in der Waldnaturschutzpolitik der deutschen Bundesländer
}

\author{
Christiane Hubo • Max Göhrs
}

Eingegangen: 15. Januar 2021 / Überarbeitet: 8. November 2021 / Angenommen: 10. November 2021 / Online publiziert: 14. Dezember 2021

(C) Der/die Autor(en) 2021

Zusammenfassung Waldnaturschutz ist ein umwelt- und klimarelevantes Politikfeld, das durch starke Konflikte zwischen Schutz- und Nutzinteressen geprägt ist. Der Beitrag untersucht anhand zentraler Konflikte, inwieweit die Tätigkeit der Landesregierungen den konfligierenden Interessen des Naturschutz- und des Forstsektors entsprechen, und welchen Einfluss unterschiedliche politische Parteien darauf haben. Damit leistet er einen empirischen Beitrag zur Parteiendifferenztheorie in einem wenig erforschten Feld.

Die Waldpolicies zwischen 2002 und 2020 wurden nach Legislaturperioden erhoben, für die jeweils bezogen auf die Sektorinteressen ein Positionswert ermittelt wurde. Der Vergleich dieser Werte erlaubt es, Politikwandel zu identifizieren. Zur Messung des Parteieneinflusses werden Regierungsbeteiligungen und Ressortzuständigkeiten herangezogen. Im Ergebnis zeigt sich, dass die Waldpolicies überwiegend forstlich orientiert sind, jedoch eine starke Tendenz Richtung Naturschutz zu verzeichnen ist. Naturschutzorientierten Politikwandel hat es in nahezu allen Bundesländern gegeben, in wenigen Fällen auch forstorientierten. Die Grünen haben entscheidend zum Politikwandel beigetragen, in geringerem Maße auch die SPD und in einigen Fällen CDU/CSU, die jedoch überwiegend für den Erhalt des Status quo eintraten. Damit ist eine deutliche Parteiendifferenz feststellbar. Die Messung des Parteieneinflusses durch die Ressortzuständigkeit hat sich für das stark durch ministerielle Kompetenzen geprägte Politikfeld als geeignet erwiesen. Die Parteizugehörigkeit der Minister*innen zeigt sich als bedeutender Faktor für die PolicyOrientierung des Regierungshandelns im Waldnaturschutz.

Keywords Forstpolitik · Ländervergleich · Naturschutzpolitik · Politikwandel · Politische Parteien

Christiane Hubo $(\bowtie) \cdot$ Max Göhrs

Professur für Forst- und Naturschutzpolitik, Georg-August-Universität Göttingen, Göttingen,

Deutschland

E-Mail: chubo@gwdg.de 


\title{
Political party difference in forest nature conservation policy of the German Bundeslaender
}

\begin{abstract}
Forest nature conservation is an environmental and climate-relevant policy field that is characterized by strong conflicts between interests on protection and utilization. Based on central conflicts, the article examines the extent to which the policy outputs of the state governments correspond to the conflicting interests of the nature conservation and forestry sectors, and what influence different political parties have on this. In doing so, he makes an empirical contribution to the partisan theory in a field that has not been well researched. The data are collected between 2002 and 2020 according to legislative periods, for each of which a position value is determined based on the interests of the sectors. The comparison of these values makes it possible to identify policy change. Government involvement and departmental responsibilities are used to measure party influence. The result shows that the policy outputs are predominantly forest-oriented, but there is a strong trend towards nature conservation. There has been a nature conservation-oriented policy change in almost all Bundeslaender, in a few cases forest-oriented ones as well, and in all the states there has also been the preservation of the status quo. The Greens have made a decisive contribution to policy change towards nature conservation interests, to a lesser extent the SPD and in some cases the CDU/CSU, which, however, predominantly advocated maintaining the status quo. This shows a clear difference between the parties. In the policy field, which is strongly characterized by ministerial competencies, the measurement of party influence through departmental responsibility has proven its worth. The party affiliation of the ministers proves to be an important factor for the policy orientation.
\end{abstract}

Schlüsselwörter Country comparison $\cdot$ Forest policy $\cdot$ Nature conservation policy $\cdot$ Policy change $\cdot$ Political parties

\section{Einleitung}

Obwohl die Parteiendifferenztheorie eine der wichtigsten Theorien zur Erklärung von Varianz in der Policy-Forschung ist (Töller 2017, S. 134; Sack und Töller 2018, S. 606), lassen sich bisher kaum allgemeingültige Aussagen darüber treffen, welchen Einfluss Parteiendifferenz auf die Umweltpolitik hat. Einer der Gründe ist darin $\mathrm{zu}$ sehen, dass bisher wenige empirische Untersuchungen vorliegen. Ein weiterer möglicher Grund ist die hohe Differenziertheit des Umweltschutzes selbst. Die verschiedenen Felder, in denen Umweltschutz eine Rolle spielt, weisen unterschiedliche Rahmenbedingungen auf, etwa hinsichtlich der Interaktion mit den jeweiligen Nutzerressorts, institutionellen Konstellationen und der öffentlichen Aufmerksamkeit. Grundlegend für einen Vergleich dieser Bedingungen, aus dem allgemeine Aussagen erschlossen werden könnten, sind empirische Studien zu den einzelnen Feldern. Während zur Parteiendifferenz im technischen Umweltschutz bereits einige Studien vorliegen (Bäck et al. 2015; Töller 2019), gibt es im „grünen Umweltschutz“ noch sehr wenig Erkenntnisse. Auch der Naturschutz weist eine Diversität nach Landnut- 
zungen wie Land- und Forstwirtschaft auf, die gesondert zu betrachten sind. Die vorliegende Studie will hierzu einen Beitrag leisten, in dem sie die Parteiendifferenz im Waldnaturschutz aufklärt.

Mit einem Anteil von $32 \%$ an der Landesfläche in Deutschland hat der Wald eine große Relevanz für den Naturschutz. Auch wenn Wald eine naturnahe Landnutzungsform ist, die ihn für Erholung und als Lebensraum für Tiere und Pflanzen besonders geeignet macht, ist der Wald in der Praxis vorrangig ein Wirtschaftsraum zur Produktion von Holz. Damit ist er Gegenstand des klassischen umweltpolitischen Konflikts zwischen Nutzungs- und Schutzinteressen. Für dieses Feld, das in den letzten Jahrzehnten weniger im Rampenlicht der politischen Öffentlichkeit stand als etwa die Energie- und die Verkehrspolitik, ist noch zu klären, wie sich die politischen Parteien positionieren, ob es Unterschiede gibt und welche Rolle insbesondere die Grünen spielen.

\section{Theoretischer Rahmen}

\subsection{Parteiendifferenz}

Die Grundannahme der Parteiendifferenztheorie ist, dass politische Parteien unterschiedliche inhaltliche Positionen vertreten, die sie auch in unterschiedliches Regierungshandeln überführen. Demnach bewirkt ein Machtwechsel durch geänderte parteipolitische Kräfteverhältnisse Änderungen in der Staatstätigkeit (Schmidt und Ostheim 2007). Die Realisierung dieses ideellen Wirkmechanismus der Parteiendemokratie wurde erstmals von Hibbs (1977) systematisch untersucht und ist seither viel diskutiert, kritisiert und modifiziert worden (z. B. Tufte 1978; Hicks und Swank 1992; Zohlnhöfer 2003; Wenzelburger 2015; Töller 2017). Dabei wurde deutlich, dass verschiedene Faktoren Parteieffekte behindern oder überlagern können.

Die untersuchte Parteiendifferenz bezieht sich regelmäßig auf Unterschiede zwischen dem linken und dem rechten Parteienspektrum, das sich auf der klassischen sozio-ökonomischen Konfliktlinie abbilden lässt (Bräuninger und Debus 2012; Downs 1957). Auf dieser lässt sich die Umweltpolitik, deren Ausweitung als Ausdruck eines Wandels von materialistischen zu postmaterialistischen Werten interpretiert worden ist (Inglehart 2008), nicht ohne weiteres verorten (Carter 2013). Entsprechend widersprüchlich sind die Ergebnisse einzelner Studien, von denen einige zwar eine unterschiedliche Parteiprogrammatik, jedoch keine Auswirkung auf das Regierungshandeln feststellten (Seeger 2003; Wurster 2010). Andere haben Parteiendifferenzen im umweltpolitischen Regierungshandeln nachweisen können (Volkery 2008; Knill et al. 2010a; Bäck et al. 2015; Töller 2017), die jedoch unterschiedliche Themenkomplexe abdecken und entsprechend widersprüchlich sind. Es erscheint daher sinnvoll, nach einzelnen Umweltbereichen zu unterscheiden. Eine weitere Lösung könnte sein, die unterschiedlichen Dimensionen der Umweltpolitik getrennt zu betrachten. So lässt sich eine Werte- und eine instrumentelle Dimension unterscheiden, wobei letztere der sozio-ökonomischen Konfliktlinie zuordbar ist. Linksorientierte, einschließlich sozialdemokratischer und grüner Parteien präferieren auch in der Umweltpolitik Eingriffe in das Wirtschaftsleben, die von liberalen und zum Teil auch 
konservativen Parteien abgelehnt werden (Töller 2012, 2017, S. 135 f.), was sich in einer eher marktbasierten Umweltpolitik der rechteren Parteien niederschlägt. Die Wertedimension wird hingegen abgebildet in der sozio-kulturellen Konfliktlinie, die zwischen Tradition und Progression, Materialismus und Postmaterialismus verläuft (Inglehart 1997; Bender et al. 2014; Decker 2018). Diese Wertedimension führt da$\mathrm{zu}$, dass christliche Parteien dem Umweltschutz näherstehen als die an der Sicherung von Arbeitsplätzen orientierten Sozialdemokraten (Knill et al. 2010a, S. 304; Bäck et al. 2015). Da sich diese Konfliktlinien seit den 1960er-Jahren stark pluralisiert haben (Decker 2018, S. 43 ff..), müssten für jeden Sektor der Umweltpolitik zunächst die konkreten Inhalte der instrumentellen und der Wertedimension ermittelt werden, um Parteiendifferenzen erkennen zu können. Für den Waldnaturschutz hat sich gezeigt, dass CDU/CSU und FDP eine Tendenz zum Marktliberalismus und zur Holznutzung haben, während alle linkeren Parteien, inklusive der Grünen, eine stärkere Ausrichtung zum Naturschutz aufweisen, verbunden mit einer klaren Verortung pro Sozialstaatlichkeit (Göhrs und Hubo 2019).

Als eine entscheidende institutionelle Einflussgröße des Regierungshandelns ist die Ressortverteilung identifiziert worden (Martin und Vanberg 2004). Allerdings besteht über die Bedeutung für die Parteiendifferenz in der Umweltpolitik noch wenig Klarheit (Töller 2019, S. 583). Das Dilemma der Umweltministerien zwischen größerer Profilierungsfähigkeit durch ein eigenes Haus und dem Verzicht auf innerministerielle Einfluss- und Unterstützungsmöglichkeiten in integrierten Häusern (Müller-Brandeck-Bocquet 1996; Pehle 1998) führt dazu, dass Parteien, die umweltpolitische Ziele erfolgreich in Regierungshandeln umsetzen wollen, einerseits die Besetzung des Umweltressorts anstreben müssen (Linhard und Rabe 2015, S. 162; Pappi et al. 2018, S. 325). Da wichtige Weichenstellungen in den themenrelevanten Nutzer- oder Wirtschaftsministerien entschieden werden, müssten sie jedoch auch die Besetzung dieser Ministerposten anstreben. Die Grünen konnten bei einer Regierungsbeteiligung regelmäßig das Umweltressort besetzen, selten jedoch gelang es ihnen, auch relevante Nutzungsressorts zu erhalten. Anders verhält sich dies in der Waldnaturschutzpolitik, hier war das relevante Forstressort, das häufig mit dem Naturschutz in einem Ministerium angesiedelt ist, für die Grünen in vielen Fällen erreichbar.

\subsection{Politikwandel}

Die Analyse von Parteiendifferenz ist vor allem deshalb bedeutsam, weil die Parteiendifferenz eine wichtige Voraussetzung ist, um in der Parteiendemokratie Politikwandel herbeizuführen. Die gesellschaftliche Pluralität und ihre Veränderungen finden ideeller Weise durch die Parteien Eingang in die politischen Entscheidungsprozesse. Die Wirkung der Parteiendifferenz lässt sich somit am Ausmaß von Politikwandel nach solchen Regierungswechseln, die zugleich Parteienwechsel sind, messen. Entsprechend kann die Parteiendifferenz als ein Erklärungsfaktor für Politikwandel herangezogen werden, wenn dieser nach Regierungswechseln eingetreten ist (Jenkins-Smith et al. 2014).

Politikwandel wird in der Literatur unterschiedlich definiert, wobei es vielfach um Beschreibungen des Wandlungsprozesses geht (so etwa bei Lindblom 1969), jedoch 
auch um die inhaltliche Dimension (Howlett 2011). Als anknüpfungsfähig erweist sich der Ansatz von Knill et al. (2010b), der Politikwandel präzisiert als „Politikergebniswandel“" und damit auf die Gesamtheit aller legislativen Tätigkeiten fokussiert (ähnlich auch Hall 1989 und Jenkins-Smith et al. 2014). Der Ansatz von Knill et al. bezieht sich explizit auf die Erfassung von Politikwandel in bestimmten Politikfeldern. Im Anschluss an Knill verstehen wir unter Politikwandel im Politikfeld Waldnaturschutz Veränderungen von Politikinhalten in verbindlichen Programmen, die Aussagen zum Naturschutz im Wald treffen. Der Wandel von Politikinhalten lässt sich ablesen an den Änderungen von Programmzielen und -instrumenten im Zeitablauf. Von den drei Ebenen, auf denen sich Politikwandel entfaltet (Knill und Tosun 2012, S. 28; Jann und Wegrich 2014), betrachten wir vorrangig Veränderungen auf der Programmebene, also des policy outputs, die unmittelbar politischen Prozessen und Entscheidungen zuordenbar sind, auch wenn damit noch nichts über die tatsächliche Wirkung gesagt ist, die Programmänderungen bei den Adressaten bewirken (policy outcome) und in der erfahrbaren Lebenswelt erzeugen (policy impact). Reale Veränderungen im Wald, insbesondere der Wald-Biodiversität, müssen hier unberücksichtigt bleiben.

\subsection{Forschungsfragen und Hypothesen}

Aus dem bisherigen Forschungsstand ergeben sich keine klaren Parteiendifferenzen, jedoch relative Tendenzen. Demnach bewirkt die Beteiligung der Grünen an der Regierung häufiger eine Veränderung zugunsten des Naturschutzes als die Regierungsbeteiligung anderer Parteien, linke Parteien setzen sich stärker für Umweltschutz und rechte Parteien stärker für Nutzungsinteressen ein. Mit Blick auf den Waldnaturschutz stellt sich nun die Frage, inwieweit dieser Befund für dieses Feld gilt und welche Besonderheiten des Waldnaturschutzes ggf. abweichende Parteieneffekte begründen könnten.

Die Waldnaturschutzpolitik wird stark durch Verordnungen und Erlasse auf Ministerebene geprägt. Daher vermuten wir das größte Machtpotential im Waldnaturschutz bei den zuständigen Ministerien bzw. den Ressorts Forstwirtschaft und Naturschutz, die meistens im Landwirtschafts- bzw. Umweltministerium angesiedelt sind. Anzunehmen ist, dass die von linken Parteien geführten Forst- und Naturschutzressorts eher schutzorientierte Policy Outputs fördern (H1). Zwar können die Interessen von Arbeitnehmern im Konflikt mit Umweltinteressen stehen, doch hat die Verhinderung der Ausbeutung der Natur ein großes Gewicht und soll sozial gerecht gestaltet werden.

Anzunehmen ist weiterhin, dass die Besetzung der Ministerposten durch rechte Parteien eher zu nutzungsorientierten Policy Outputs führt, sofern diese Parteien sich als eigentümer- und wirtschaftsfreundlich definieren. Andererseits fügt sich Naturschutz in den Heimatschutz als Thema rechter Parteien wie auch in das Weltbild christlich orientierter Parteien, die der Bewahrung der Natur als Schöpfung Gottes eine Bedeutung beimessen. Dies begründet die Annahme, dass rechte und christliche Parteien auch naturschutzorientiert handeln, soweit Wirtschaftsinteressen dadurch nicht gefährdet werden $(\mathrm{H} 2)$. 
Grüne Parteien können zwar sowohl links als auch rechts angesiedelt sein, da jedoch Umweltschutz ihr Kernthema ist, kann von ihnen grundsätzlich die größte Unterstützung für Schutzinteressen erwartet werden (H3). Weniger klar ist dies bei Konflikten zwischen Naturschutz und erneuerbaren Energien, da energiepolitische Themen für die Grünen traditionell einen besonders hohen Stellenwert haben, während sie dem Naturschutz lange Zeit distanziert gegenüberstanden. Relevant wird dies bei der Aufstellung von Windenergieanlagen im Wald und der energetischen Verwendung von Holz. Dieses Themenfeld überschreitet den Konflikt zwischen Forstwirtschaft und Naturschutz und bleibt einer gesonderten Untersuchung vorbehalten.

Es ist also wahrscheinlich, dass es für den Waldnaturschutz einen Unterschied macht, welche Parteien die Regierung stellen. Der vorliegende Beitrag untersucht die Parteiendifferenz, indem Unterschiede im Regierungshandeln in der Waldnaturschutzpolitik aufgezeigt und die Ergebnisse mit den jeweiligen Regierungsparteien korreliert werden. Damit soll geklärt werden, ob Politikwandel im Waldnaturschutz zumindest teilweise auf den Einfluss von Parteien zurückzuführen ist. Einflüsse durch weitere Faktoren sind möglich, doch soll hier zunächst der Parteienfaktor geklärt werden.

\section{Methodik}

Das Regierungshandeln in der Waldnaturschutzpolitik messen wir anhand der Policy Outputs zum Waldnaturschutz in den Bundesländern, im Folgenden als ,Waldpolicies" bezeichnet; sie sind unsere abhängige Variable. Das Handeln der politischen Parteien, die jeweils die Regierung gebildet haben, ist unsere erklärende Variable. Durch Korrelation dieser Variablen suchen wir nach Anhaltspunkten für eine Beziehung zwischen beiden.

\subsection{Waldpolicies}

Für die Erhebung der Policy Outputs zum Waldnaturschutz sind die Themen sowie der zeitliche und räumliche Rahmen festzulegen. Weiterhin ist zu klären, wie die Varianz gemessen und auf welche Weise Politikwandel erfasst wird.

\subsubsection{Auswahl und Erhebung der Waldpolicies}

Inhaltlich fokussieren wir die Waldpolicies auf zentrale Streitthemen des Waldnaturschutzes. Zur Erfassung der Konfliktlage hat sich das Konzept der Politiksektoren bewährt, in denen sich unterschiedliche Positionen kristallisieren (Hubo und Krott 2010). „Politiksektor“ bezeichnet eine funktional abgegrenzte politische Arena, in der empirisch beobachtbare Gruppen staatlicher und nichtstaatlicher Akteure bezogen auf einen bestimmten Regelungsgegenstand interagieren (Verbij 2008, S. 23). In der Forstpolitikforschung gibt es eine lange Tradition, den Politiksektor als Bezugsrahmen für die Politikanalyse zu verwenden (Rayner et al. 2001). Der Waldnaturschutz ist ein sektorenübergreifendes Politikfeld, das den ökologischen 
Aspekt der Waldbewirtschaftung in der Zuständigkeit des Forstwirtschaftssektors, und zugleich den Wald als Teil der natürlichen Umwelt in der Zuständigkeit des Naturschutzsektors umfasst. Grundlegend sind Forstwirtschaftsakteure traditionell an der Aufrechterhaltung der herkömmlichen Forstwirtschaft bzw. einer Stärkung der ökonomischen Orientierung interessiert (Brukas und Weber 2009; Peters und Schraml 2014), während Naturschutzakteure einen Wandel hin zu einer stärkeren Berücksichtigung der biologischen Vielfalt einfordern (SRU 2012, Tz. 377).

Am deutlichsten kristallisiert sich der Konflikt in der von Naturschutzakteuren angestrebten Flächensegregation, welche das integrative Leitbild der multifunktionalen Forstwirtschaft auf einem kleinen Teil der Gesamtfläche verlässt. Sie beinhaltet die Überlassung bestimmter Flächen für die natürliche Waldentwicklung ohne forstliche Bewirtschaftung, während die übrigen Flächen als Wirtschaftswald genutzt werden. Im Wirtschaftswald dreht sich der Konflikt um die Ausrichtung der forstlichen Bewirtschaftung auf ökologische Ziele (integrativer Naturschutz), insbesondere den Umbau zu Mischwäldern und die Verwendung heimischer Baumarten. Aus Naturschutzsicht wird die Kombination beider Ansätze, Segregation und Integration, befürwortet (SRU 2012 Tz. 383).

Der grundlegende Konflikt in Bezug auf die Verfolgung von Naturschutzzielen auf separaten Flächen, zeigt sich seit ca. 100 Jahren in Verbindung mit Naturschutzgebieten, insbesondere mit Nationalparken als der Kategorie für Großschutzgebiete mit den strengsten Vorgaben. Aktuell reflektiert die Diskussion um natürliche Waldentwicklung (Meyer et al. 2011) diesen Konflikt. In den Bundesländern gibt es einige Aktivitäten für Zielformulierungen und instrumentelle Umsetzungen, etwa durch Biodiversitätsstrategien oder weitere Nationalparke.

In Bezug auf den Ansatz der Integration von Naturschutzzielen in die forstliche Bewirtschaftung liegen die wichtigsten Kontroversen in den Themen struktur- und artenreiche Mischwälder, gebietsfremde Baumarten und Schutz heimischer Arten durch das Schutzgebietsnetz Natura 2000 (SRU 2012; BfN 2021). Politikwandel existiert in allen drei Themen: die Waldumbauprogramme, die in den Ländern schon in den 1970er-Jahren aufgelegt wurden, haben mit Blick auf Anpassungen an den Klimawandel eine neue Aktualität bekommen (Storch und Winkel 2012). Für den Konflikt um die Wahl von gebietsfremden versus heimischen Baumarten gibt es unterschiedliche Instrumente in den Bundesländern. Weitere Relevanz hat das Thema durch die EU-Verordnung über die Prävention und das Management der Einbringung und Ausbreitung invasiver gebietsfremder Arten (VO Nr. 1143/2014) bekommen. Bei den Natura 2000-Gebieten, deren Ausweisung seit den 1990er-Jahren im Prozess ist, geht es aktuell um die rechtliche Sicherung der Gebiete, die Erstellung von Managementplänen und finanzielle Förderungen (BfN 2021). In den Bundesländern wird diese Thematik unterschiedlich gehandhabt und instrumentell ausgestaltet.

Auf Grund dieser Konfliktlage wählen wir als Streitthemen zum integrativen Ansatz die Zertifizierung des Landeswaldes nach dem FSC-Standard, mit der die integrativen Ziele eingefordert werden, und als besonders strittigen Punkt den Umgang mit gebietsfremden Baumarten. Als Streitthemen zur Flächensegregation wählen wir die Sicherung von Natura 2000-Gebieten und die Überlassung von Waldflächen für die natürliche Entwicklung (NWE). 
Wir untersuchen Policy Outputs zwischen 2002 und 2020 und decken damit die politische Hochkonjunktur dieser Themen ab, auch wenn die Themen teilweise schon früher relevant waren. Die deutsche Sektion des von internationalen Umweltverbänden gegründeten Forest Stewardship Council (FSC) hat sich 1997 gegründet und 2001 den ersten Zertifizierungsstandard erlassen (Geilhof et al. 2019). Etwa ab 2003/2004 wurde das Thema invasive gebietsfremde Arten im Rahmen der Vorbereitungen für die Nationale Biodiversitätsstrategie politisch aktuell (Hubo et al. 2007). 2004 hat die Europäische Kommission die Gebietsvorschlage für Natura 2000 bestätigt und die Sicherung dieser Gebiete verlangt. Die Überlassung von Flächen für die natürliche Waldentwicklung, die bereits seit den 1990er-Jahren von Umweltverbänden gefordert wird (Hubo und Krott 2015), ist mit dem sog. 5\%-Ziel in die Nationalen Strategie für Biologische Vielfalt der Bundesregierung von 2007 eingegangen (BMU 2007).

In räumlicher Hinsicht ermitteln wir die Policy Outputs von allen Bundesländern mit Ausnahme des Stadtstaates Bremen, da Bremen kaum über nennenswerte Waldanteile verfügt und entsprechend auch keine öffentliche Forstverwaltung hat.

Um eine Zuordnung zu den Regierungsparteien zu ermöglichen, verwenden wir als Basiseinheit für die Analyse Legislaturperioden. Für den Untersuchungszeitraum ergeben sich 53 Legislaturperioden, für die wir Waldpolicies erheben. Dafür haben wir in den Ländern Regierungsprogramme, Wald- und Naturschutzgesetze, themenrelevante Verordnungen und Erlasse, Waldbaurichtlinien, Richtlinien für die forstliche Förderung und Waldberichte ausgewertet. Überwiegend waren diese online recherchierbar, teilweise mussten sie von den Ministerien angefordert werden.

\subsubsection{Policy-Positionen}

Die Unterschiede zwischen den erhobenen Waldpolicies messen wir anhand von Policy-Positionen, die sich danach unterscheiden lassen, ob sie eher den Interessen des Naturschutzsektors oder denen des Forst- und Holzwirtschaftssektors entsprechen. Als Anhaltspunkte für die Interessen verwenden wir Aussagen von Fachverbänden des Naturschutzes und der Forst- und Holzwirtschaft und leiten daraus PolicyPositionen ab.

Unsere Analyse zeigt, dass es eine Policy-Position des Forstsektors gibt, die allen untersuchten Streitthemen gemeinsam ist. Diese besteht in der Befürwortung der freien Entscheidung des Waldeigentümers und ist als eine liberale Position auf der Staat-Markt-Konfliktlinie (Göhrs und Hubo 2019) zu verorten. Der freiheitlichen Position entspricht nicht nur die Ablehnung staatlicher Reglementierung, die über allgemeine Vorgaben oder die Sicherung der Freiheitsrechte hinaus geht, sondern durchaus auch eine Akzeptanz von freiwilligen Naturschutzleistungen, für die finanzielle Anreize gegeben werden. Die themenübergreifende Policy-Position des Naturschutzsektors besteht im Gegensatz dazu in der Steuerung der forstlichen Bewirtschaftung durch verbindliche Vorgaben des Staates, die im Staatswald leichter durchsetzbar sind als im Privatwald, so dass für letzteren auch eine Steuerung durch finanzielle Anreize befürwortet wird. Daraus lässt sich nicht schließen, dass es bei den Streitthemen keine inhaltlichen Divergenzen, die auf der sozio-kulturellen Wertedimension zu verorten sind, gäbe, und nur um den Weg gestritten würde. Die 
Inhalte des FSC-Standards, der Verzicht auf gebietsfremde Baumarten, die forstlichen Konsequenzen einer Natura 2000 Gebietssicherung und die Stilllegung forstlicher Flächen stoßen auch sachlich auf heftigen Widerstand im Forstsektor. Diese von Naturschützern angestrebten Inhalte wären ohne staatliche Regulierung kaum durchsetzbar, da sie den forstlichen Interessen entgegengesetzt sind. Die Konflikte kulminieren somit in den Policy-Positionen pro und contra staatliche Regulierung, hinter denen jedoch auch inhaltliche Konflikte stehen.

Um die Policy-Positionen für einen Vergleich zugänglich zu machen, verorten wir sie auf einer Viererskala. Dabei werden Policy-Positionen des Forstsektors mit 0 und die des Naturschutzsektors mit 1 kodiert, Zwischenstufen sind 0,33 und 0,66. Damit bewerten wir, welcher Policy-Position die Outputs am Ende einer Legislaturperiode entsprechen. Legislaturperioden, in denen der bisherige Stand beibehalten wurde, in der es also keine Änderung in die eine oder andere Richtung gab, erhalten den gleichen Wert wie die vorherige Legislaturperiode, da dies den Policy-Positionen unverändert entspricht. Aus den Werten zu den vier Streitthemen wird für jede Legislaturperiode das arithmetische Mittel gebildet, wobei solche Legislaturperioden nicht berücksichtigt wurden, in denen nur ein Streitthema relevant war. Für jede dieser Legislaturperioden gewinnen wir so einen Wert für die Policy-Orientierung.

\subsubsection{Vermessung der Waldpolicies}

Mit dem so gewonnenen Maßstab lassen sich die einzelnen Streitthemen wie folgt vermessen:

Wenn die FSC-Zertifizierung im Landeswald eingeführt wurde, so geschah dies meistens mehr oder weniger flächendeckend (in 8 von 15 Ländern, Geilhof et al. 2019). Auch wenn die Zertifizierung durch eine nicht-staatliche Organisation vorgenommen wird, enthält diese Situation den Faktor staatliche Regulierung, da den Landesforsten diese Zertifizierungsform von der Regierung bzw. dem zuständigen Ministerium vorgegeben wird. Dem liegt eine naturschutzorientierte Policy-Position zugrunde, die mit 1 kodiert wird. In drei Ländern wurde nur ein geringer Flächenanteil (weniger als 10\%) zertifiziert, womit sich eher die forstliche Policy-Position durchgesetzt hat, so dass wir mit 0,33 kodieren. Wurde FSC gar nicht im Landeswald eingeführt (4 Länder), wurden alle Legislaturperioden mit 0 kodiert.

Beim Streit um gebietsfremde Baumarten besteht das Forstinteresse in der Nutzung derselben. Dies lässt sich dadurch verwirklichen, dass die Baumartenwahl des Eigentümers in dieser Hinsicht nicht eingeschränkt wird, d. h. dass es entweder keine Regelung dazu gibt oder eine, die die Nutzung ermöglicht. In beiden Fällen kodieren wir die Legislaturperiode mit 0. Wurde hingegen ein Policy-Output geschaffen, der geringfügige Einschränkungen beinhaltet, wobei also noch relativ viel Entscheidungsspielraum beim Eigentümer bleibt, verwenden wir den Score 0,33. Dies ist der Fall, wenn ein „hinreichender“ oder ,überwiegender“ Anteil heimischer Baumarten auf der Fläche enthalten sein soll, oder Förderzuschläge z. B. bei der ausschließlichen Verwendung heimischer Baumarten gezahlt werden. Mit 0,66 wird kodiert, wenn es stärkere Einschränkungen gibt, wie z. B. die Vorgabe, dass nicht-heimische Baumarten einen Anteil von 5\% nicht überschreiten dürfen, oder der Ausschluss nicht-heimischer Baumarten aus der Förderung in Schutzgebieten. Eine totale Be- 
schränkung z. B. durch ein Verbot bestimmter nicht-heimischer Baumarten bzw. die Vorgabe, nur heimische Baumarten anzupflanzen, sowie der generelle Ausschluss aus der Förderung erhalten die Kodierung 1.

Bei der Sicherung von Natura 2000-Gebieten besteht die Policy-Position des Naturschutzes darin, die Gebiete unter den Schutz eines naturschutzrechtlichen Regelungsregimes zu stellen, was am stärksten durch die Ausweisung als Naturschutzgebiete gelingen kann, etwas weniger stark durch Landschaftsschutzgebiete. Allerdings verschwimmen die Unterschiede in der konkreten Anwendung, so dass wir hier nicht unterscheiden. Wir kodieren diese Fälle mit 1, sofern im jeweiligen Bundesland die Schutzgebiete mit starken Einschränkungen geschützt werden, und mit 0,66 bei geringen Einschränkungen. Die forstliche Policy-Position beinhaltet vorzugsweise keine Gebietssicherung nach Naturschutzrecht, oder eine Sicherstellung erforderlicher Maßnahmen durch Vertragsnaturschutz, was wir mit 0 kodieren. Wird anstelle eines naturschutzrechtlichen Schutzregimes eine landesweite Verordnung, allgemein oder mit gebietskonkreten Vorgaben (Leben 2018), erlassen oder finanzieller Ausgleich gewährt, kodieren wir mit 0,33 .

Bei der Überlassung von Waldflächen für eine natürliche Entwicklung besteht die Policy-Position des Naturschutzes darin, 5 oder mehr Prozent der Waldfläche insbesondere im Landeswald dafür auszuweisen, und zwar wiederum gesichert durch eine naturschutzrechtliche Schutzkategorie (BUND 2011). Beiträge in diese Richtung kodieren wir mit 1, wenn das 5\%-Ziel erreicht wurde, und mit 0,66, wenn der Anteil noch deutlich unter $5 \%$ liegt. Die forstliche Policy-Position sieht das 5\%-Ziel unter Berücksichtigung naturschutzrechtlich nicht gesicherter Flächen als längst erreicht

Tab. 1 Kodierung der Waldpolicies nach Positionen des Forst- (0) und des Naturschutzsektors (1)

\begin{tabular}{|c|c|c|c|c|}
\hline Issue & 0 & 0,33 & 0,66 & 1 \\
\hline$F S C$ & Keine Flächen & Weniger als $50 \%$ & Mindestens $50 \%$ & Flächendeckend \\
\hline \multirow[t]{2}{*}{$\begin{array}{l}\text { Gebiets- } \\
\text { fremde } \\
\text { Baumar- } \\
\text { ten }(B A)\end{array}$} & Keine Vorgaben & $\begin{array}{l}\text { Hinreichender oder } \\
\text { überwiegender } \\
\text { Anteil heimischer } \\
\text { BA vorgegeben }\end{array}$ & $\begin{array}{l}\text { Max. } 5 \% \text { nicht- } \\
\text { heimische BA }\end{array}$ & $\begin{array}{l}\text { Vorgabe, nur hei- } \\
\text { mische BA an- } \\
\text { zubauen/Verbot } \\
\text { gebietsfremder BA }\end{array}$ \\
\hline & $\begin{array}{l}\text { Vorgaben, die } \\
\text { gebietsfremde } \\
\text { BA ermöglichen }\end{array}$ & $\begin{array}{l}\text { Förderanreize für } \\
\text { heimische BA }\end{array}$ & $\begin{array}{l}\text { Ausschluss nicht- } \\
\text { heimischer BA aus } \\
\text { der Förderung in } \\
\text { Schutzgebieten }\end{array}$ & $\begin{array}{l}\text { Genereller Aus- } \\
\text { schluss nicht- } \\
\text { heimischer BA aus } \\
\text { der Förderung }\end{array}$ \\
\hline \multirow[t]{2}{*}{$\begin{array}{l}\text { Sicherung } \\
\text { von } \\
\text { Natura } \\
\text { 2000- } \\
\text { Gebieten }\end{array}$} & Keine Sicherung & $\begin{array}{l}\text { Landesweite VO } \\
\text { statt Schutzgebiete } \\
\text { nach Naturschutz- } \\
\text { recht }\end{array}$ & $\begin{array}{l}\text { Weniger strenge } \\
\text { Schutzgebiete mit } \\
\text { allenfalls geringen } \\
\text { Einschränkungen }\end{array}$ & $\begin{array}{l}\text { Strenge } \\
\text { Schutzgebiete mit } \\
\text { deutlichen } \\
\text { Einschränkungen }\end{array}$ \\
\hline & $\begin{array}{l}\text { Sicherung durch } \\
\text { Verträge }\end{array}$ & $\begin{array}{l}\text { Finanzieller Aus- } \\
\text { gleich }\end{array}$ & $\begin{array}{l}\text { der forstlichen } \\
\text { Bewirtschaftung }\end{array}$ & $\begin{array}{l}\text { der forstlichen } \\
\text { Bewirtschaftung }\end{array}$ \\
\hline \multirow[t]{2}{*}{$\begin{array}{l}5 \% \\
\text { natürliche } \\
\text { Waldent- } \\
\text { wicklung } \\
\text { (NWE5) }\end{array}$} & $\begin{array}{l}\text { Rücknahme von } \\
\text { Flächen aus dem } \\
\text { Schutzstatus in } \\
\text { die Nutzung }\end{array}$ & $\begin{array}{l}\text { Finanzieller Aus- } \\
\text { gleich für die Ent- } \\
\text { nahme von Flächen } \\
\text { aus der Nutzung }\end{array}$ & $\begin{array}{l}\text { NWE durch Ge- } \\
\text { bietsschutz nach } \\
\text { Naturschutzrecht }\end{array}$ & $\begin{array}{l}\text { NWE durch Ge- } \\
\text { bietsschutz nach } \\
\text { Naturschutzrecht }\end{array}$ \\
\hline & $\begin{array}{l}\text { Keine Entnahme } \\
\text { neuer Flächen } \\
\text { aus der Nutzung }\end{array}$ & $\begin{array}{l}\text { NWE durch forstli- } \\
\text { che Schutzgebiete }\end{array}$ & $\begin{array}{l}\text { Jedoch noch deut- } \\
\text { lich unter } 5 \% \text { der } \\
\text { Waldflächen }\end{array}$ & $\begin{array}{l}\text { Mindestens } 5 \% \\
\text { der Waldflächen }\end{array}$ \\
\hline
\end{tabular}


an und lehnt die als „Stilllegung“ bezeichnete Entnahme weiterer Waldflächen aus der Nutzung ab, insbesondere, wenn die Flächen unter Naturschutz gestellt werden (DFWR 2015). Wir kodieren mit 0, wenn Flächen aus dem Schutzstatus entlassen und wieder in die forstliche Nutzung genommen wurden, oder wenn keine neuen Flächen entnommen wurden. Für den Forstsektor ist es zwar nicht erstrebenswert, aber durchaus akzeptabel, wenn die Flächenentnahme finanziell entgolten wird. Diesen Fall, wie auch die Ausweisung von Flächen als forstliches Schutzgebiet, die der Forstsektor Schutzgebieten nach Naturschutzrecht vorzieht, kodieren wir mit 0,33. Anhand dieser Kriterien (Tab. 1) werden die Policy Outputs zu den vier Streitthemen für jede einzelne Legislaturperiode in den Ländern bewertet.

\subsubsection{Politikwandel}

Der Vergleich der Perioden zeigt nicht nur die Unterschiede in der Orientierung der Waldpolicies, sondern ermöglicht auch, Politikwandel zu identifizieren. Um den Wandel zu erfassen, vergleichen wir die Policy-Orientierung jeder Periode mit der vorherigen innerhalb eines Landes. Dabei reduziert sich die Anzahl der Legislaturperioden auf 38 (53 abzüglich 1 Periode pro Land), da jeweils die Veränderung bzw. der Status quo der Policy-Orientierung gegenüber der vorherigen Periode gewertet wird. Um zu deutlicheren Ergebnissen zu kommen, bilden wir eine Skala von 0 (Forst) bis 1 (Naturschutz) mit vier Quartilen. Ist der Wert unverändert, betrachten wir dies als Aufrechterhaltung der vorherigen Policy-Position, was wir als Status quo (SQ) bezeichnen. Hierbei unterscheiden wir, in welchem Quartil der Status quo aufrechterhalten wurde. Verändert sich der Wert um weniger als 0,25, ist dies eine geringe Änderung, ab 0,25 sprechen wir von einer mittleren und ab 0,5 von einer größeren Veränderung.

\subsection{Parteiendifferenz}

Zum Einflusspotential der Parteien tragen die Regierungsbeteiligung, die Zuständigkeit für die Ministerien und die Stärke im Parlament bei (vgl. Martin und Vanberg 2004). Allerdings korrelieren diese Parameter stark miteinander. Die Sitzverteilung im Parlament ist eine entscheidende Grundlage für die Bildung einer Regierung und für die Durchsetzung von Ressortansprüchen. Die Ministerämter wiederum werden in Koalitionsverhandlungen verteilt. Laver und Shepsle (1990, S. 874) bezeichnen Kabinettsmitglieder als Regierung in many respects, Policy Outputs werden stark von der Parteicouleur des entsprechenden Ministers geprägt.

Wir messen die Parteiendifferenz zunächst durch Regierungsbeteiligung. Primär ermöglicht diese Aussagen über Koalitionsregierungen, die hier jedoch nicht weiter untersucht werden, zugleich lässt sie aber auch Aussagen darüber zu, welche Politik die einzelnen Parteien ermöglichen und mitverantworten. Die Ressortzuständigkeit erlaubt eine genaue Zuordnung zu der jeweiligen Partei und wird als Ergänzung hinzugezogen. Die Linke und die FDP haben nur selten ein relevantes Ressort geleitet, so dass zu diesen beiden Parteien wenig Aussagen möglich sind.

Relevant ist primär das Forstministerium, dass durch Richtlinien und Erlasse auf die Zertifizierung des Staatswaldes, die Baumartenwahl und die Ausweisung von 
Flächen für die natürliche Waldentwicklung (NWE) Einfluss nehmen kann. Das Naturschutzministerium ist in der Regel für die Sicherung der Natura 2000-Gebiete zuständig und verfügt auch über Kompetenzen zur naturschutzrechtlichen Sicherung von NWE-Flächen. Beide Ministerien sind häufig in einem integrierten Ministerium zusammengefasst, weshalb wir die Begriffe Forst- und Naturschutzressort verwenden.

\section{Waldpolicies}

In $62 \%$ der Legislaturperioden waren die Waldpolicies stärker forstlich $(0-0,5)$ und in $38 \%$ stärker naturschutzorientiert (Abb. 1).

Innerhalb des Untersuchungszeitraums hat sich dieses Verhältnis jedoch gewandelt. Die Waldpolicies der Legislaturperioden, die in der ersten Hälfte (2002-2010) begonnen haben, waren noch deutlich stärker forstlich- als naturschutzorientiert $(0,26)$. In den Perioden, die ab 2011 begonnen haben, war der Output dagegen im Durchschnitt öfter naturschutz- als forstlich-orientiert (Abb. 2). Diese Entwicklung fand in allen vier Streitthemen statt, am ausgeprägtesten bei der natürlichen Waldentwicklung. Bei den gebietsfremden Baumarten blieb der Output auch im zweiten Zeitraum stärker forstlich orientiert. Da die Grundtendenz bei allen Streitthemen gleich ist, begrenzen wir die weitere Analyse auf die Durchschnittswerte.

Von den 38 Perioden, die auf Politikwandel untersucht wurden, weisen 18 einen Status quo gegenüber der vorherigen Periode auf, jeweils 9 (24\%) mit Forst- und mit Naturschutzorientierung. In 17 Perioden (45\%) gab es eine Veränderung Richtung Naturschutz und nur in 3 Perioden (8\%) Richtung Forst (Abb. 3).

Bezogen auf die Länder bedeuten die Ergebnisse, dass über den gesamten Zeitraum in 11 Ländern die Forstorientierung $(0-0,5)$ der Waldpolicies überwiegt, wäh-

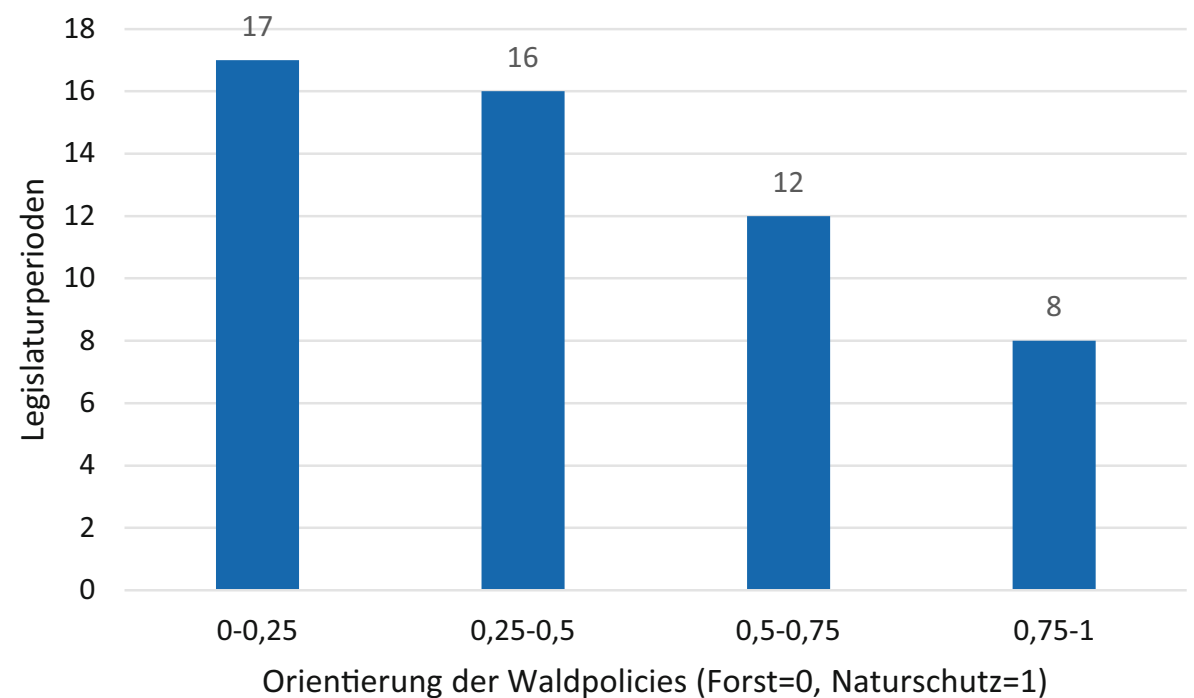

Abb. 1 Orientierung der Waldpolicies in den Ländern 2002-2020 $(n=53)$ 


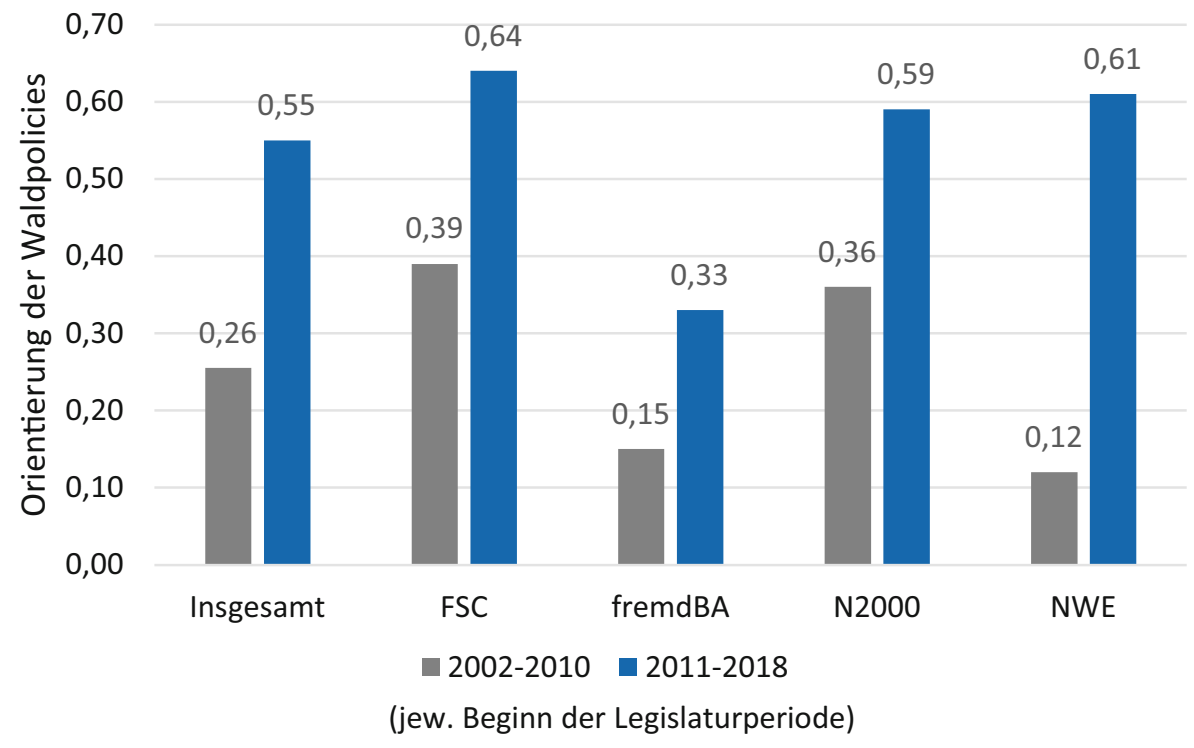

Abb. 2 Entwicklung der Orientierung der Waldpolicies nach 2 Zeiträumen $(n=53)$

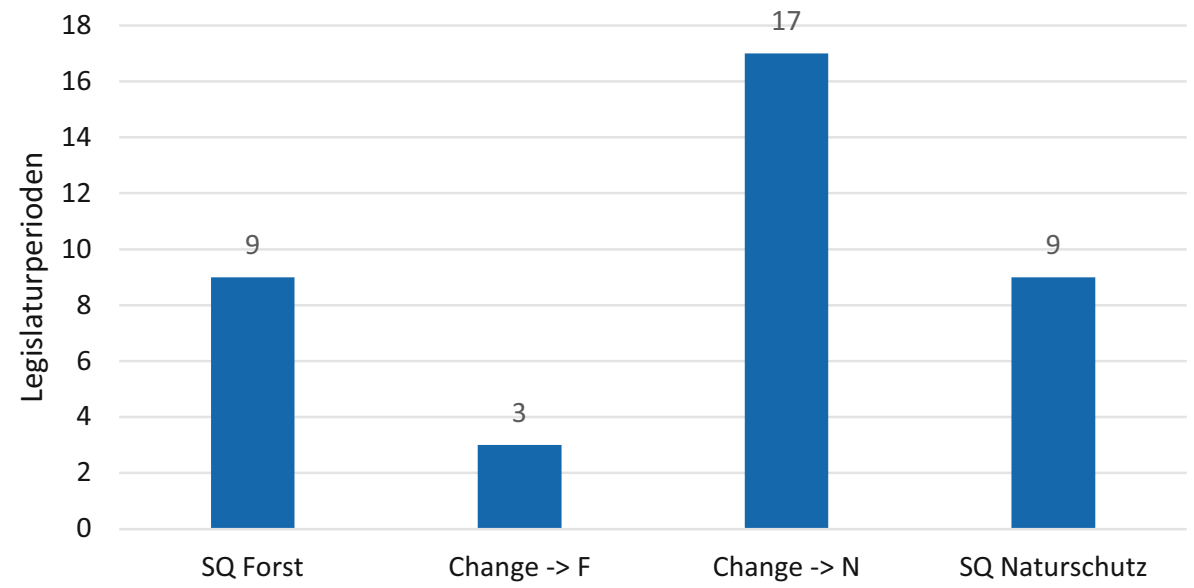

Abb. 3 Politikwandel im Waldnaturschutz $(n=38)$

rend in vier Ländern der Output stärker naturschutzorientiert $(0,5-1)$ ist (jew. Mittelwert der Legislaturperioden, s. Tab. 2).

Politikwandel Richtung Naturschutz fand in fast allen Ländern statt, wenn auch in unterschiedlichem Ausmaß. So gab es in 3 Ländern (Sachsen, Thüringen, Bayern) nur sehr geringe Bewegungen in diese Richtung, in 11 Ländern jedoch größere Schritte. In 3 Ländern ging die Entwicklung anschließend wieder Richtung Forstorientierung. In allen Ländern gab es mindestens eine Periode ohne Änderung, in 7 Ländern als forst- und in 6 Ländern als naturschutzorientierter Status quo. Abgesehen von Mecklenburg-Vorpommern, wo im gesamten Beobachtungszeitraum eher 


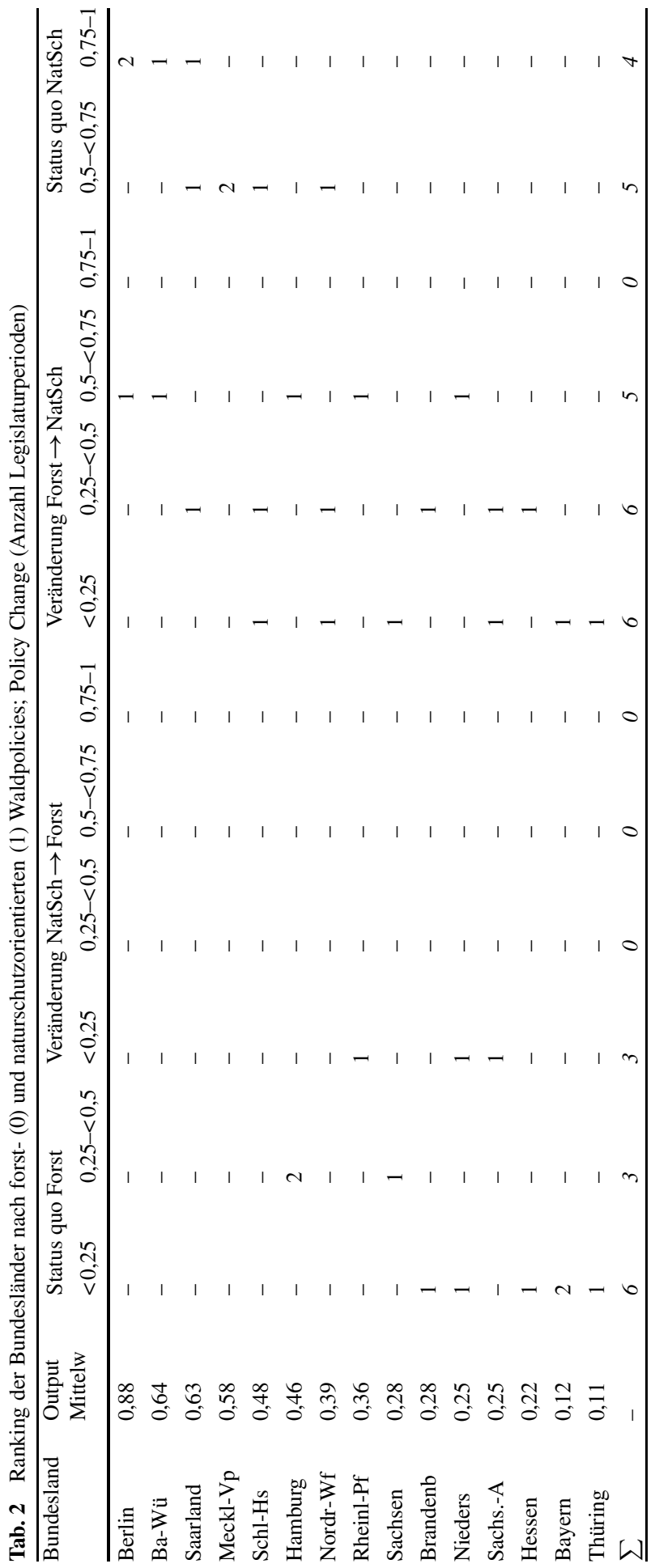


naturschutzorientierte Waldpolicies festzustellen waren, gab es somit innerhalb der Länder mindestens einen Wechsel. Zumindest diese Dynamik kann nicht auf weitgehend konstante Variablen wie der Waldanteil, Besitzverhältnisse, holzverarbeitende Industrie oder die Stärke von Umweltverbänden zurückgeführt werden.

Zusammenfassend ist festzuhalten:

1. Die Waldpolicies sind insgesamt stärker forst- als naturschutzorientiert, jedoch fand innerhalb des Untersuchungszeitraums eine Verschiebung Richtung Naturschutzinteressen statt.

2. In 14 von 15 Ländern hat Politikwandel Richtung Naturschutz stattgefunden; in nur 3 Ländern gab es eine Kehrtwende Richtung Forstwirtschaft. In allen Ländern gab es auch forstlichen oder naturschutzorientierten Status quo.

\section{Parteiendifferenzen}

\subsection{Policy Orientierung}

In den 53 untersuchten Legislaturperioden waren die Unionsparteien mit $70 \%$ am häufigsten beteiligt, gefolgt von der SPD mit 58\%. Die Grünen waren an $30 \%$ der Regierungen beteiligt, die FDP an $25 \%$ und die Linke an $11 \%$. Die Grünen haben während ihrer Regierungsbeteiligungen am stärksten zu naturschutzorientierten, die FDP am stärksten zu forstorientierten Waldpolicies beigetragen. Auch CDU/CSU waren klar stärker an forstorientieren Outputs beteiligt, währen sich SPD und Linke im Mittelfeld bewegen (Abb. 4).

Eine deutlichere Parteiendifferenz zeigt sich, wenn der Parteieneinfluss durch die Zuständigkeit für das Forst und das Naturschutzressort gemessen wird (Abb. 5).

Mit Blick auf die beiden Teilzeiträume 2002-2010 und 2011-2018 (jeweils Beginn der Legislaturperioden) wird deutlich, dass sich das Kräfteverhältnis der Parteien stark verschoben hat: starken Zunahmen bei Grünen und SPD stehen starke Abnahmen bei CDU/CSU und FDP gegenüber. Die Zahl der Regierungsbeteiligungen der Linken ist in beiden Zeiträumen gleich gering (Abb. 6).

Dieses Muster zeigt sich auch bei der Entwicklung der Ressortzuständigkeiten, wobei der Bedeutungsverlust der CDU noch deutlicher wird (Abb. 7).

Die Zunahme der naturschutzorientierten und die Abnahme der forstorientierten Waldpolicies (Abb. 2) geht also einher mit einer Zunahme der Regierungsbeteiligungen und Ressortzuständigkeiten von Grünen und SPD und einer Abnahme jener von CDU/CSU und FDP. Doch zeigt die Aufschlüsselung der Regierungsbeteiligungen nach den beiden Zeiträumen, dass die Entwicklung Richtung Naturschutz, abgesehen von den Linken, bei allen Parteien feststellbar ist, bei den rechten Parteien sogar stärker als bei SPD und Grünen (Abb. 8).

Auch die Analyse der Ressortzuständigkeit zeigt, dass unter Unionsführung zwar die Forstorientierung erhalten geblieben ist, Unions-geführte Ministerien jedoch ebenso zu einer Verschiebung Richtung Naturschutz beigetragen haben, wie Grünund SPD-geführte Ministerien (Abb. 9). 
0,70

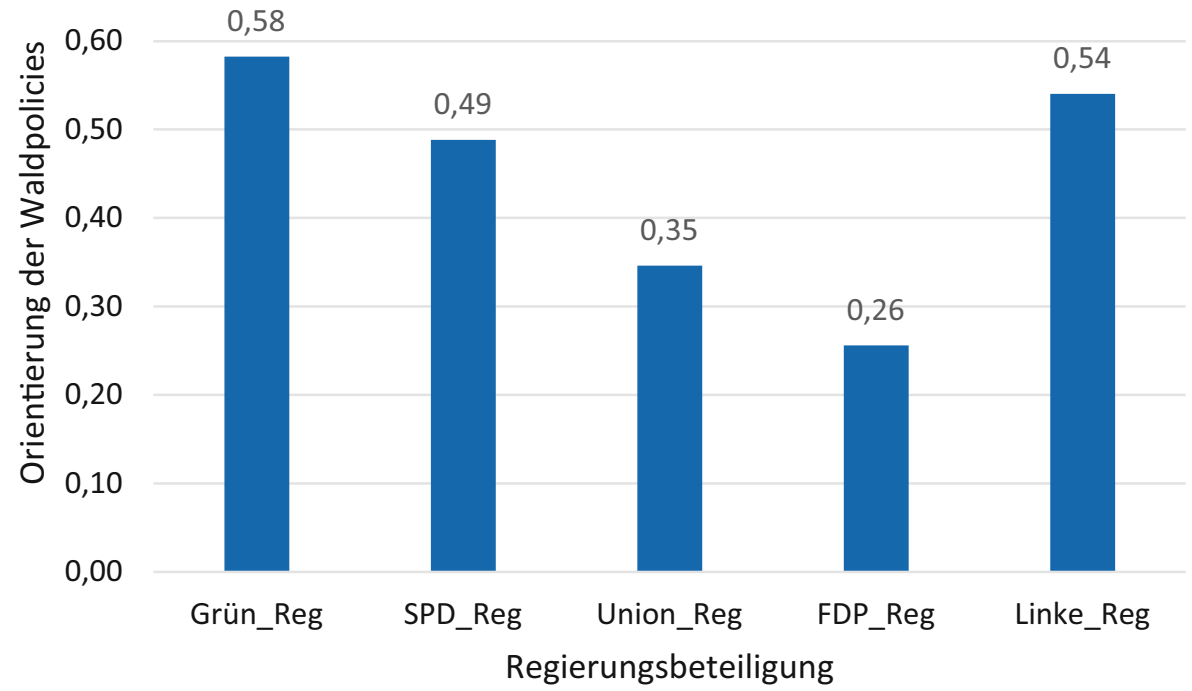

Abb. 4 Orientierung der Waldpolicies (Forst = 0, Naturschutz = 1) nach Regierungsbeteiligung $(n=53)$

0,70

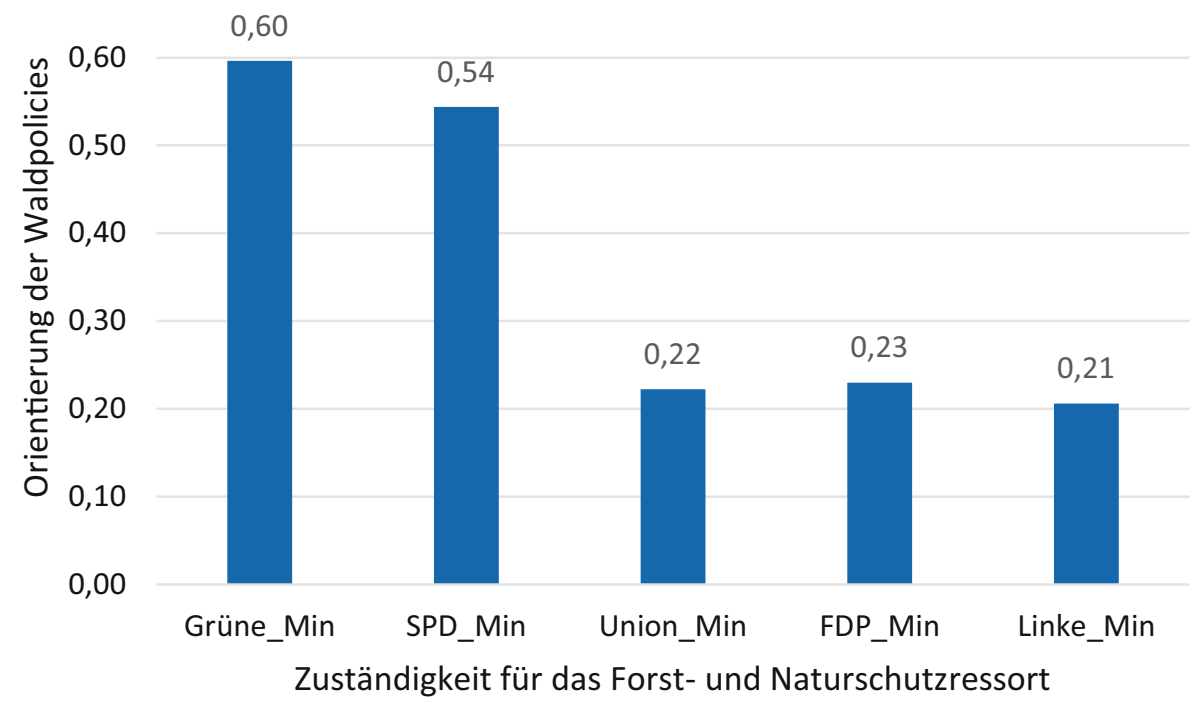

Abb. 5 Orientierung der Waldpolicies (Forst $=0$, Naturschutz $=1)$ nach Ressortzuständigkeit $(n=53)$ 


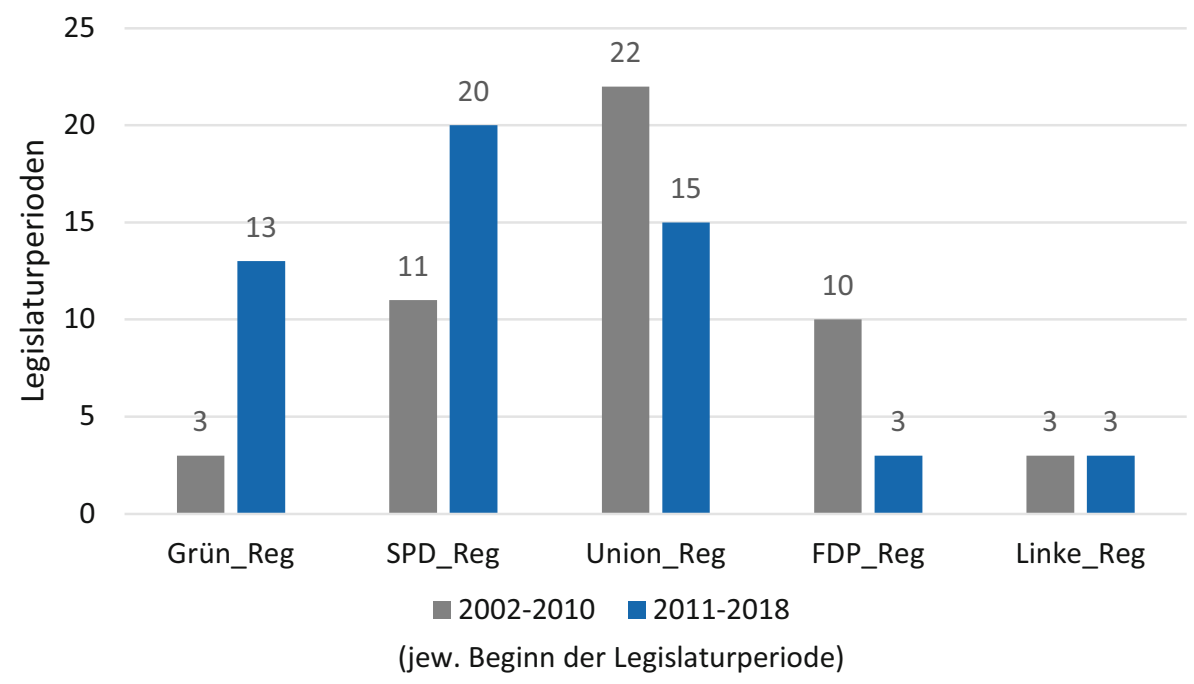

Abb. 6 Entwicklung der Regierungsbeteiligungen $(n=53)$

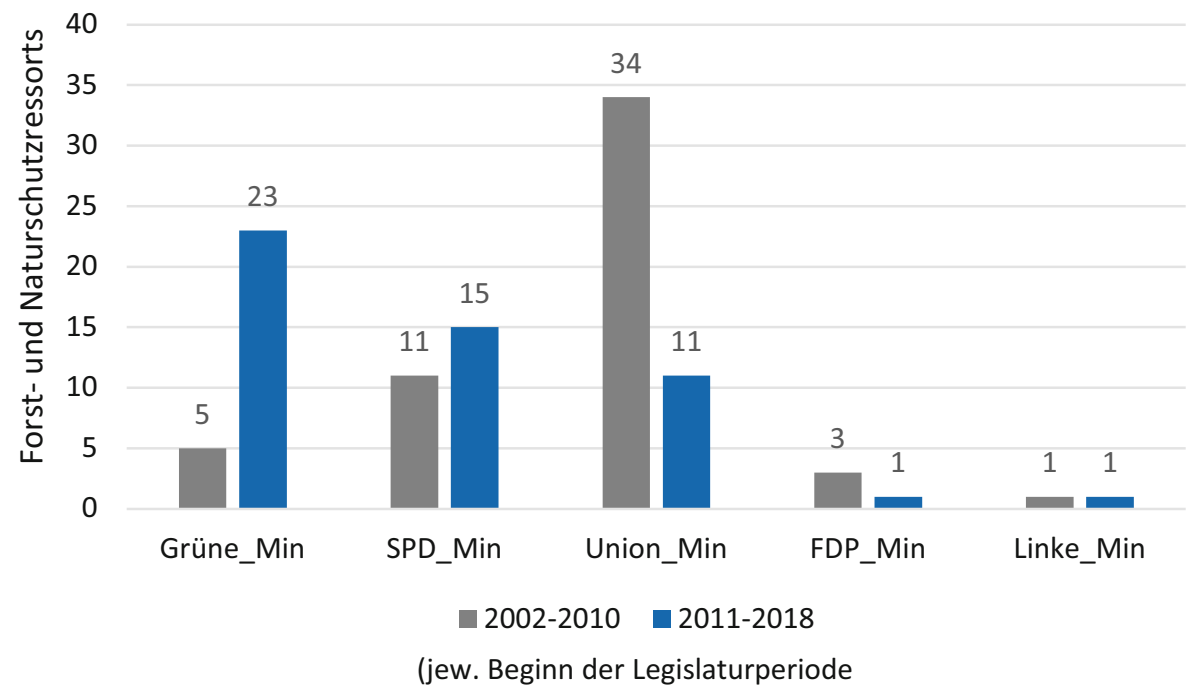

Abb. 7 Entwicklung der Ressortzuständigkeit $(\mathrm{n}=53)$ 


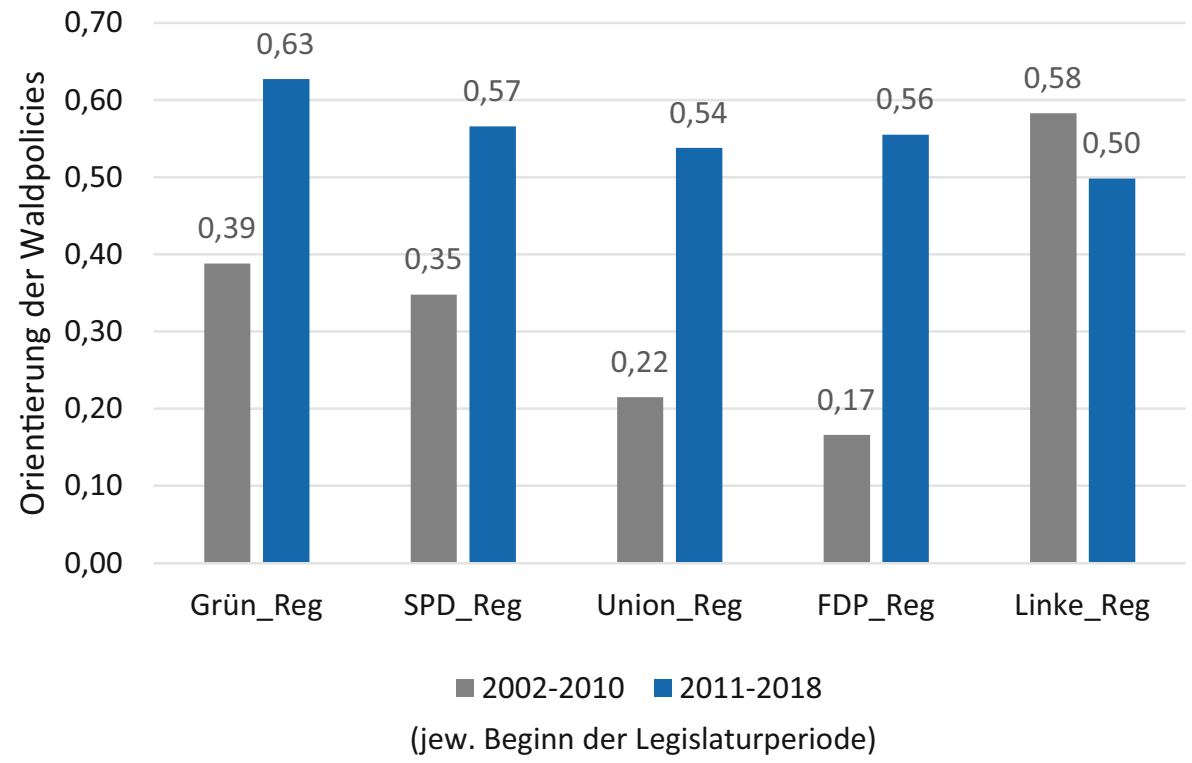

Abb. 8 Entwicklung der Orientierung der Waldpolicies (Forst=0, Naturschutz $=1$ ) nach Regierungsbeteiligung $(n=53)$

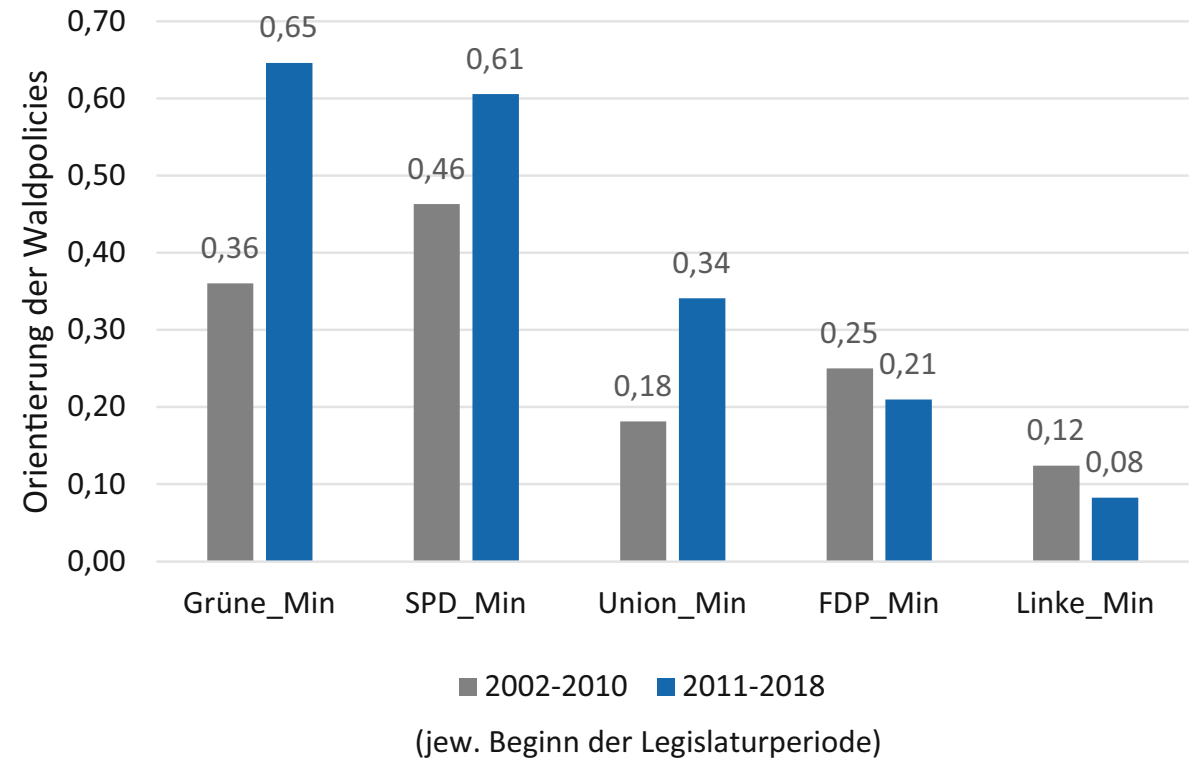

Abb. 9 Entwicklung der Orientierung der Waldpolicies (Forst = 0, Naturschutz $=1$ ) nach Ressortzuständigkeit $(n=53)$ 


\subsection{Politikwandel}

Der Vergleich der Regierungsbeteiligungen beim Politikwandel ergibt, dass nahezu alle Parteien zu jeder Kategorie in mindestens einer Legislaturperiode beigetragen haben. Herausragend ist die Beteiligung von SPD und Grünen an Veränderungen Richtung Naturschutz (Abb. 10).

Zieht man die Ressortzuständigkeit in die Betrachtung ein, relativiert sich der Einfluss der SPD auf naturschutzorientierten Politikwandel, die große Bedeutung von Minister*innen der Grünen wird deutlich (Abb. 11).

Eine detailliertere Analyse des naturschutzorientierten Wandels zeigt, dass stärkerer Wandel nur dann zustande kam, wenn beide Ressorts durch Grüne oder SPD geführt wurden. Unter CDU/CSU-Führung kam es häufig zum Erhalt des forstlichen Status quo, doch war auch geringer naturschutzorientierter Wandel möglich. Forstorientierter Wandel fand nur dann statt, wenn eine der rechteren Parteien das Forstressort führte (Abb. 12 und 13).

Zusammenfassend ist festzuhalten:

1. Die Entwicklung der Waldpolicies zu mehr Naturschutzorientierung geht einher mit einer Zunahme grünen und sozialdemokratischen Parteieneinflusses. Die Entwicklung zu stärkerer Naturschutzorientierung hat nicht nur unter der Ressortverantwortung von Grünen und SPD stattgefunden, sondern ebenso unter CDU/CSURessortverantwortung. Dabei blieben unter letzterer die Policy Outputs insgesamt stärker forstorientiert.

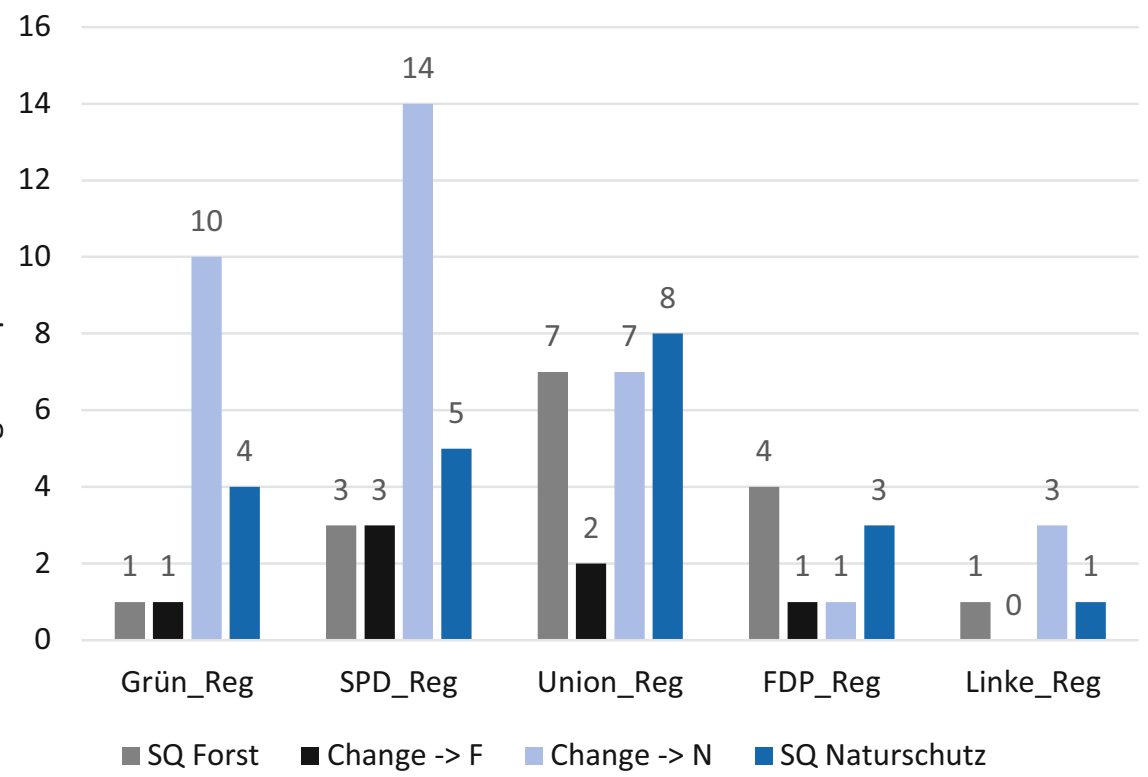

Abb. 10 Beteiligung der Regierungsparteien am Politikwandel $(n=38)$ 


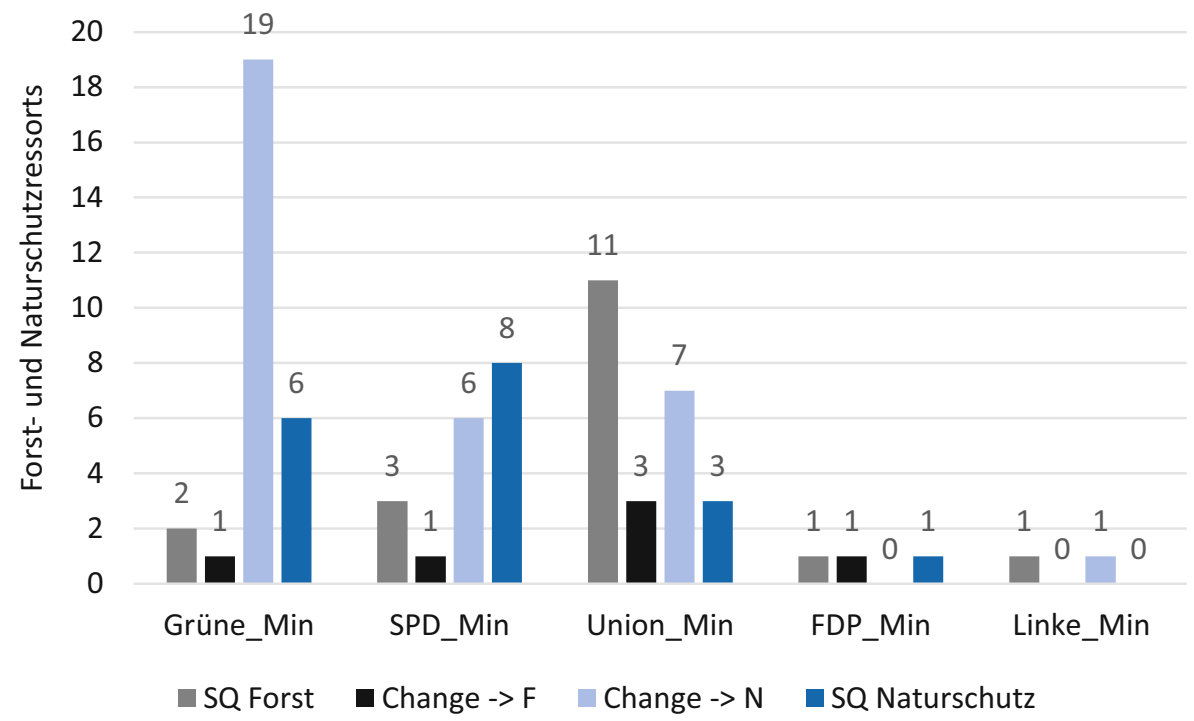

Abb. 11 Ressortzuständigkeit beim Politikwandel $(n=38)$

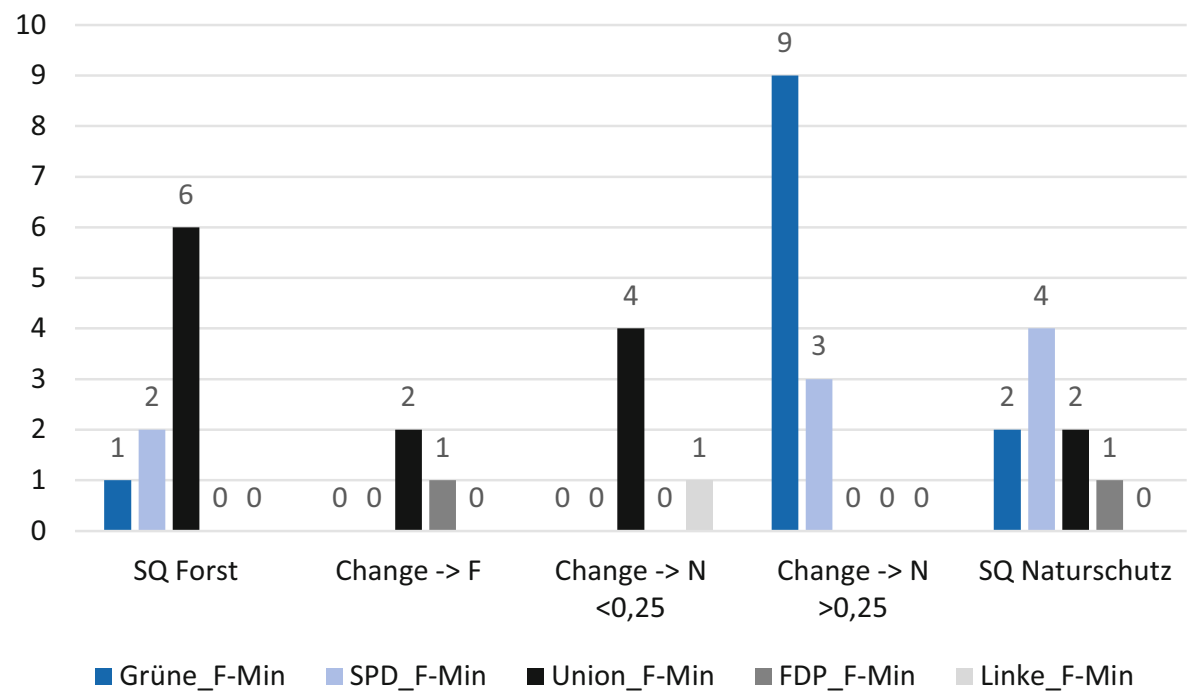

Abb. 12 Politikwandel nach Zuständigkeit für das Forstressort $(n=38)$

2. Während die Analyse des Politikwandels nach Regierungsbeteiligungen ergibt, dass Grüne und mehr noch die SPD in herausragendem Umfang an naturschutzorientiertem Wandel beteiligt waren, ansonsten jedoch keine bedeutenden Parteiendifferenzen festzustellen sind, zeigt die Analyse nach Ressortzuständigkeiten eine deutliche Parteiendifferenz, insbesondere den überragenden Einfluss der Grünen auf naturschutzorientierten Wandel. 


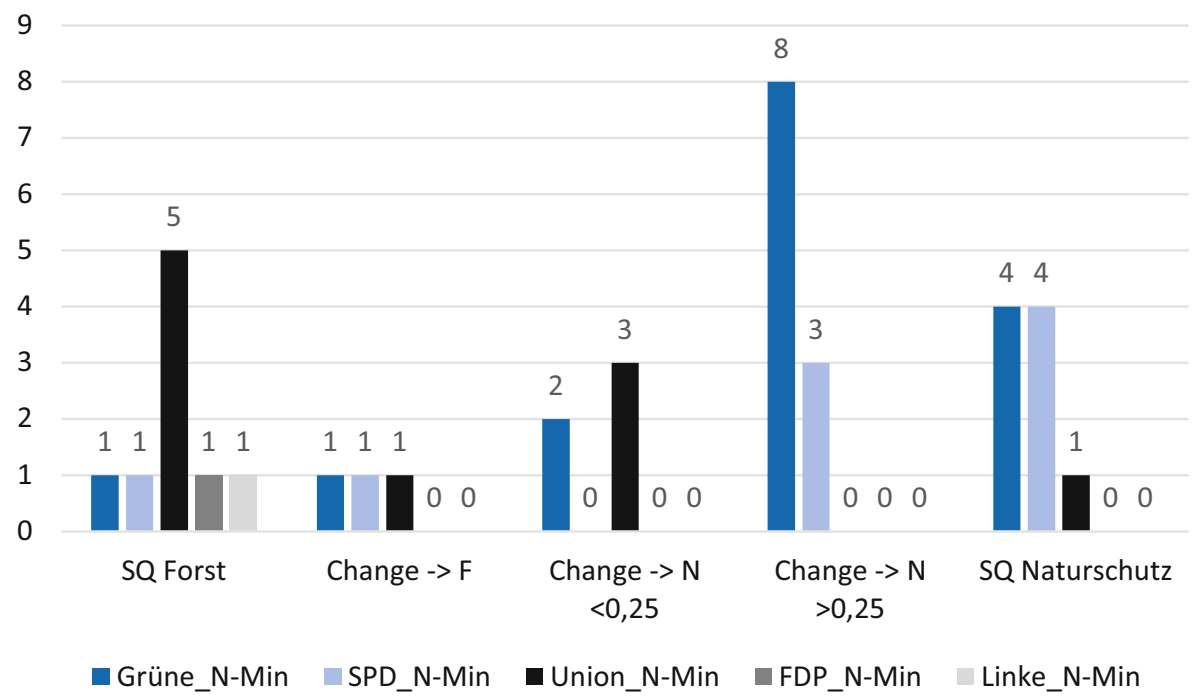

Abb. 13 Politikwandel nach Zuständigkeit für das Naturschutzressort $(n=38)$

\section{Ergebnisdiskussion}

Festzuhalten ist, dass die Messung des Parteieneinflusses durch die Regierungsbeteiligung eine geringere Parteiendifferenz zeigt, als wenn die Ressortzuständigkeit herangezogen wird, die genauere Zuordnungen zu einer Partei erlaubt. Diese Messung wird im Weiteren zugrunde gelegt.

Der Parteieneinfluss steht in folgendem Verhältnis zur an den Interessen des Forst- und des Naturschutzsektors gemessenen Varianz der Waldpolicies in den Bundesländern: Grüne und SPD haben, wenn sie beide Ressorts innehatten, doppelt so oft naturschutz- wie forstorientierte Waldpolicies und damit den größten Teil der naturschutzorientierten Waldpolicies erzeugt. CDU/CSU haben, wenn sie beide Ressorts zu verantworten hatten, ganz überwiegend forstorientiert gehandelt und damit den größten Teil der forstorientierten Waldpolicies erzeugt. Auch wenn nur das Forstressort in CDU/CSU-Hand lag, waren die Waldpolicies überwiegend forstorientiert. Bemerkenswert ist weiterhin, dass eine Ressortverantwortung der Linken mit forstorientierten Waldpolicies einhergeht (Tab. 3).

Die getrennte Analyse von zwei Zeiträumen hat gezeigt, dass es bei den Waldpolicies eine Dynamik Richtung Naturschutz gibt, die einher geht mit einer Machtverschiebung von den rechteren zu den linkeren Parteien. Zugleich hat sich, gemessen an den Policy Outputs, die Policy-Orientierung von Grünen, SPD und CDU/CSU Richtung Naturschutz verschoben. Dies spricht dafür, dass andere Faktoren als die Parteien diese Dynamik beeinflusst haben. Möglicherweise hat sich die CDU, getrieben von den Stimmengewinnen der Grünen in Verbindung mit eigenen Stimmenverlusten, zu mehr Naturschutz anstecken lassen, doch kann dies bestenfalls eine Teilerklärung sein, da ja auch die Grünen und die SPD sich Richtung mehr Naturschutz bewegt haben. Eine Rolle könnte spielen, dass die EU verstärkt die 
Tab. 3 Forst- und naturschutzorientierte Waldpolicies und Politikwandel nach Ressortzuständigkeit (Anzahl Legislaturperioden)

\begin{tabular}{|c|c|c|c|c|c|c|c|c|c|c|}
\hline \multicolumn{2}{|c|}{ Ressort } & \multicolumn{3}{|c|}{$\begin{array}{l}\text { Waldpolicy Orien- } \\
\text { tierung }\end{array}$} & \multicolumn{2}{|c|}{ Politikwandel } & \multirow[b]{2}{*}{$\mathrm{F} \rightarrow \mathrm{N}<0,25$} & \multirow[b]{2}{*}{$\mathrm{F} \rightarrow \mathrm{N}>0,25$} & \multirow[b]{2}{*}{$\begin{array}{l}\text { SQ } \\
\text { NatSch }\end{array}$} & \multirow[b]{2}{*}{$\sum$} \\
\hline Forst & NSch & Forst & NatSch & $\sum$ & $\begin{array}{l}\text { SQ } \\
\text { Forst }\end{array}$ & $\mathrm{N} \rightarrow$ Forst & & & & \\
\hline Grüne & & 4 & 8 & 12 & 1 & - & 1 & 8 & 2 & 12 \\
\hline SPD & & 4 & 8 & 12 & 1 & - & - & 3 & 4 & 8 \\
\hline $\mathrm{CDU} / \mathrm{C}$ & SSU & 18 & 2 & 20 & 5 & 1 & 3 & - & 1 & 10 \\
\hline $\mathrm{CDU}$ & FDP & 2 & 0 & 2 & 1 & - & - & - & - & 1 \\
\hline CSU & $(\mathrm{FW})$ & 1 & 0 & 1 & - & - & 1 & - & - & 1 \\
\hline $\mathrm{CDU}$ & SPD & 1 & 0 & 1 & - & 1 & - & - & - & 1 \\
\hline $\mathrm{CDU}$ & Grüne & 0 & 1 & 1 & - & - & - & - & 1 & 1 \\
\hline FDP & Grüne & 1 & 1 & 2 & - & 1 & - & - & 1 & 2 \\
\hline Linke & Grüne & 1 & 0 & 1 & - & - & 1 & - & - & 1 \\
\hline SPD & Linke & 1 & 0 & 1 & 1 & - & - & - & - & 1 \\
\hline$\sum$ & & 33 & 20 & 53 & 9 & 3 & 6 & 11 & 9 & 38 \\
\hline
\end{tabular}

Umsetzung naturschutzorientierter Vorgaben einfordert (Natura 2000) und nationale Impulse (NWE5-Ziel) eine Wirkung entfaltet haben. Eine nicht unerhebliche Rolle dürfte auch der Einfluss von Verbänden gespielt haben, was noch zu untersuchen sein wird.

Die Analyse des Politikwandels erschließt eine weitere Innensicht auf die Dynamik in der Waldnaturschutzpolitik (Tab. 3). Wenn Grüne und SPD jeweils beide Ressorts innehatten, haben sie fast durchgehend naturschutzorientierte Politik betrieben, die Grünen ganz überwiegend in Form von stärkerem Wandel Richtung Naturschutz. Eine Ausnahme stellt für beide Parteien der Stadtstaat Hamburg dar. Die bundesweit erste schwarz-grüne Koalition auf Landesebene in Hamburg ab 2008 war spannungsreich. Wir haben keine Hinweise auf ein Engagement der Grünen in der Waldnaturschutzpolitik, obwohl hier die Grünen das Naturschutz- und das Forstressort verantwortet haben. In der folgenden Legislaturperiode hat sich unter der SPD an dem Status quo der eher forstlichen Orientierung nichts geändert. Erst unter Rot-Grün in der folgenden Periode wurden starke Veränderungen Richtung Naturschutz eingeleitet. Somit hat sich 2008 in der Koalition der Grünen mit der CDU im Waldnaturschutz noch kein grüner Effekt gezeigt, der später aber in der Koalition mit der SPD möglich wurde.

CDU/CSU haben, wenn sie für beide Ressorts oder nur für das Forstressort zuständig waren, in den meisten Fällen den forstlichen Status quo bewahrt oder einen Wandel Richtung Forst bewirkt. Es gab jedoch auch einige Fälle mit leichten Änderungen Richtung Naturschutz und in Koalition mit den Grünen die Bewahrung des naturschutzorientierten Status quo.

Für die Linke wird deutlich, dass es innerhalb des forstorientierten Outputs auch eine leichte Bewegung Richtung Naturschutz gegeben hat, als die Grünen das Naturschutzressort führten (in Thüringen 2014). In Brandenburg hat eine rot-rote Regierung ab 2009 den starken forstlichen Status quo aufrechterhalten. In der folgenden Periode wurde unter der gleichen Regierungskonstellation eine Änderung Richtung 
Naturschutz eingeleitet, die somit nicht durch einen Parteieneffekt erklärbar scheint. Doch hat 2009 die SPD nur das Forstressort geleitet, die Linke das Naturschutzressort, während 2014 die SPD beide Ressorts bekommen hat. Die Änderung Richtung Naturschutz fand bei dem Streitthema Natura 2000 statt, das in der Zuständigkeit des Naturschutzministeriums liegt. In beiden Fällen, in denen die Linke ein Ministerium geführt hat, in Thüringen und Brandenburg, erweist sie sich als eher forstund holznutzungsorientierte Partei.

Unter Zugrundelegung aller Zuständigkeiten für die beiden Ressorts in den 53 Legislaturperioden (ergibt $53 * 2=106-1$ für die Freien Wähler=105 Fälle) bestätigt die Analyse der Waldpolicies die Eingangshypothesen nur schwach. Der Anteil der naturschutzorientierten Waldpolicies bleibt insbesondere bei den Grünen und weniger stark bei der SPD als Mitte-Links-Partei hinter den Erwartungen zurück, doch haben beide Parteien sich deutlich mehr für den Waldnaturschutz eingesetzt, als die anderen Parteien. Auch bei den christlichen Parteien wäre ein etwas höherer Anteil naturschutzorientierter Waldpolicies erwartbar gewesen und bei der FDP als rechtsliberaler Partei ein höherer Anteil forstorientierter Politik. Bei letzterer lässt die geringe Fallzahl allerdings kaum belastbare Aussagen zu, ebenso wie bei der Linken, die mit $100 \%$ eher forstorientierter Politik ebenfalls überrascht hat (Tab. 4).

Deutlich bestätigt dagegen die Analyse des Politikwandels in den 38 Legislaturperioden (ergibt $38 * 2=76$-1 für die Freien Wähler=75 Fälle) die Eingangshypothesen für die Parteien, die öfter Ressortverantwortung übernommen hatten. Wenn der forstorientierte Status quo und Veränderungen Richtung Forstinteressen als Forstorientierung zusammengefasst werden und entsprechend bei der Naturschutzorientierung verfahren wird, kommen die Grünen auf der Skala von 0 (Forst) bis 1 (Naturschutz) auf den Wert 0,9, die SPD auf 0,8 und CDU/CSU auf 0,4. Die Grünen zeigen also eine sehr starke Veränderungskraft für den Naturschutz, die SPD in etwas abgeschwächter Form, und die CDU hat einen Fokus auf der Bewahrung des forstlichen Status quo, ist jedoch auch zu leichten Änderungen bereit. Bei der Linken beruht die naturschutzorientierte Legislaturperiode auf einer leichten Änderung, die dem grünen Partner zuzuordnen ist, so dass für die Linke insgesamt festzustellen ist, dass sie der Eingangshypothese, nach der linke Parteien eher naturschutzorientierte Politik betreiben, nicht entspricht. Dies mag damit zusammenhängen, dass die Linke ein stärkeres Augenmerk auf den Erhalt forstlicher Arbeitsplätze im ländlichen Raum gelegt hat. Bei der FDP ist die naturschutzorientierte Legislaturperiode ein

Tab. 4 Verhältnis der Ressortzuständigkeit zur Orientierung der Waldpolicies und zum Politikwandel

\begin{tabular}{lllllllll}
\hline Parteien & \multicolumn{2}{l}{ Hypothesen } & \multicolumn{2}{l}{ Orientierung Waldpolicies } & \multicolumn{2}{l}{ Politikwandel } \\
& Forst & NSch & Ressorts & $\begin{array}{l}\text { Forst } \\
(\%)\end{array}$ & $\begin{array}{l}\text { NSch } \\
(\%)\end{array}$ & Ressorts & $\begin{array}{l}\text { Forst } \\
(\%)\end{array}$ & $\begin{array}{l}\text { NSch } \\
(\%)\end{array}$ \\
\hline Grüne & - & ++ & 28 & 36 & 64 & 28 & 11 & 89 \\
Linke & - & + & 2 & 100 & 0 & 2 & 50 & 50 \\
Mitte-links & - & + & 26 & 38 & 62 & 18 & 22 & 78 \\
Rechts-christl & ++ & + & 45 & 89 & 11 & 24 & 58 & 42 \\
Rechts-liberale & ++ & - & 4 & 75 & 25 & 3 & 67 & 33 \\
$\sum$ & k. A. & k. A. & 105 & 62 & 38 & 75 & 32 & 68 \\
\hline
\end{tabular}


Status quo, zusammen mit einem grünen Partner, so dass sich insgesamt für die FDP die Eingangshypothese eher bestätigt. Für die beiden kleinen Parteien stellen diese Ergebnisse aufgrund der geringen Fallzahlen jedoch nur Annäherungen da.

\section{Schlussfolgerungen}

Die Ergebnisse der Analysen zeigen deutlich, wie sehr Aussagen zur Parteiendifferenz von der Messmethode abhängen. Die klare Parteiendifferenz im Waldnaturschutz ließ sich nur mit den Ressortzuständigkeiten erfassen, während die oft verwendeten Regierungsbeteiligungen eher tendenzielle Unterschiede zwischen den Parteien erkennen lassen. Die Ressortzuständigkeit war für das untersuchte Politikfeld besonders geeignet, weil ein großer Teil der Waldpolicies in ministerieller Verantwortung gestaltet wird. Hier stellt sich die Frage, inwieweit sich das Untersuchungsergebnis auf andere Politikbereiche, die weniger stark administrativ geprägt sind, übertragen lässt. Diese Frage stellt sich auch im Hinblick darauf, dass die betreffenden Fachminister*innen offenbar weitgehend der Linie ihrer Partei folgen konnten, unabhängig davon, in welcher Koalition sie sich befanden. Die Verwendung der Ressortzuständigkeit als Maßstab wirft somit ein Licht auf den Einfluss von Koalitionsabsprachen. Dass dieser hier nicht erkennbar ist, mag damit erklärbar sein, dass der Wald bisher eher ein politisches Randthema darstellte. Hier zeigt sich weiterer Forschungsbedarf, um die koalitionsinternen Prozesse zu erhellen.

Ein weiterer methodischer Schlüsselfaktor ist die Messung der Varianz der abhängigen Variablen. Das hier verwendete Kriterium der Sektorinteressen hat den Vorteil hoher politischer Relevanz für die betroffenen Akteure. Die Beurteilung der Interessen beinhaltet jedoch Spielräume und setzt eine genaue Kenntnis der Politiksektoren voraus.

Inhaltlich lässt das Ergebnis den Schluss zu, dass das Handeln von Minister*innen in der Waldnaturschutzpolitik stark durch ihre Parteizugehörigkeit geprägt ist. Da die naturschutzorientierten Waldpolicies methodisch bedingt mit den Interessen des Naturschutzsektors übereinstimmen, ist zu vermuten, dass es den Umweltverbänden insbesondere, jedoch nicht nur, durch die Grünen gelungen ist, Einfluss auf die Ausrichtung der Staatstätigkeit zu gewinnen. Entsprechendes gilt für die Forstwirtschaftsinteressen, die am stärksten durch CDU/CSU und FDP, jedoch auch durch die Linke in die Regierungspolitik der Länder eingebracht wurden. Dies zeigt deutlich das Dilemma des Parteienstaates, der den Einfluss auf die politische Willensbildung durch Parteien als demokratische Teilhabe postuliert, damit jedoch die Neutralität des Staatshandelns gegenüber unterschiedlichen gesellschaftlichen Interessen zu verlieren droht.

Das Fungieren der Parteien als Türöffner für Sektorinteressen ist unabhängig davon, ob die Parteien durch voteseeking oder policyseeking motiviert sind. Allerdings musste in dieser Analyse offenbleiben, warum sich die Waldpolicies im Untersuchungszeitraum so stark Richtung Naturschutzinteressen entwickeln haben, unter grüner, sozialdemokratischer und christdemokratischer Ressortverantwortung. Vieles spricht für makropolitische Einflüsse unabhängig von den Landesparteien, wie den Einfluss der EU (Natura 2000) und ein gestiegenes Interesse an Umweltthemen, 
das weit über Deutschland hinaus geht. Doch lässt sich nicht ausschließen, dass das Erstarken der Grünen, das ja selbst ein Ausdruck von gesellschaftlichem Wandel ist, andere Parteien zu wählerorientierten Richtungsänderungen veranlasst hat. Die Erforschung solcher Ansteckungseffekte in weiteren Arbeiten erscheint vielversprechend.

Die vorliegenden Befunde sind starke Indizien für den Einfluss politischer Parteien, sie lassen aber keine Aussagen darüber zu, welche politischen Handlungen einzelner staatlicher und privater Akteure zu den Waldpolicies geführt haben. Aufschluss über die politischen Entscheidungsprozesse, Einflussnahmen von Verbänden und den Erfolg von Lobbyarbeit können Tiefenanalysen in ausgewählten Fällen geben.

Danksagung Wir danken den anonymen Gutachtern und den Teilnehmenden am Workshop „Parteiendifferenz in der Umweltpolitik“ an der Universität Göttingen für ihre hilfreichen Hinweise sowie der DFG für die Förderung (Fördernummer 376182053).

Funding Open Access funding enabled and organized by Projekt DEAL.

Open Access Dieser Artikel wird unter der Creative Commons Namensnennung 4.0 International Lizenz veröffentlicht, welche die Nutzung, Vervielfältigung, Bearbeitung, Verbreitung und Wiedergabe in jeglichem Medium und Format erlaubt, sofern Sie den/die ursprünglichen Autor(en) und die Quelle ordnungsgemäß nennen, einen Link zur Creative Commons Lizenz beifügen und angeben, ob Änderungen vorgenommen wurden.

Die in diesem Artikel enthaltenen Bilder und sonstiges Drittmaterial unterliegen ebenfalls der genannten Creative Commons Lizenz, sofern sich aus der Abbildungslegende nichts anderes ergibt. Sofern das betreffende Material nicht unter der genannten Creative Commons Lizenz steht und die betreffende Handlung nicht nach gesetzlichen Vorschriften erlaubt ist, ist für die oben aufgeführten Weiterverwendungen des Materials die Einwilligung des jeweiligen Rechteinhabers einzuholen.

Weitere Details zur Lizenz entnehmen Sie bitte der Lizenzinformation auf http://creativecommons.org/ licenses/by/4.0/deed.de.

Interessenkonflikt C. Hubo und M. Göhrs geben an, dass kein Interessenkonflikt besteht.

\section{Literatur}

Bäck, Hanna, und Marc Jale Tosun Debus. 2015. Partisanship, ministers, and biotechnology policy. Review of Policy Research 32:556-575.

Bender, S., M. Bianchi, K. Hohl, A. Jüschke, J. Schoofs, und S. Steitz. 2014. Die Policy-Positionen der Parteien bei der Bundestagswahl 2013. Eine Analyse mit dem Duisburger-Wahl-Index (DWI). In Die Bundestagswahl 2013. Analysen der Wahl-, Parteien-, Kommunikations- und Regierungsforschung, Hrsg. K.-R. Korte, 165-184. Wiesbaden: VS.

Bräuninger, T., und M. Debus. 2012. Parteienwettbewerb in den deutschen Bundesländern. Wiesbaden: VS.

Brukas, V., und N. Weber. 2009. Forest management after the economic transition. At the crossroads between German and Scandinavian traditions. Forest Policy and Economics 11:586-592.

BUND [Bund für Umwelt und Naturschutz Deutschland]. 2011. Lebendige Wälder. BUNDpositionen 57.

Bundesamt für Naturschutz. 2021. Kooperation mit Nutzern beim Natura 2000-Management. https://www. bfn.de/kooperation-mit-nutzern-beim-natura-2000-management\#anchor-6381. Zugegriffen: 4. Nov. 2021.

Bundesministerium für Umwelt, Naturschutz und Reaktorsicherheit. 2007. Nationale Strategie zur Biologischen Vielfalt. Berlin: BMU.

Carter, Neil. 2013. Greening the mainstream: party politics and the environment. Environmental Politics 22:73-94. 
Decker, F. 2018. Parteiendemokratie im Wandel, 2. Aufl., Baden-Baden: Nomos.

DFWR [Deutscher Forstwirtschaftsrat]. 2015. Nutzungsfreie Waldflächen - was sagt die Bundeswaldinventur? proWald 10:22-23.

Downs, A. 1957. An economic theory of political action in a democracy. Journal of Political Economy 65:135-150.

Geilhof, Michel, Christiane Hubo, und Max Göhrs. 2019. Der Einfluss politischer Parteien auf die Zertifizierung der staatlichen Forstwirtschaft in Deutschland. Zeitschrift für Umweltpolitik und Umweltrecht 42:161-184.

Göhrs, Max, und Christiane Hubo. 2019. Politische Parteien als Bündnispartner für den Waldnaturschutz. Allgemeine Forst- und Jagdzeitung 190:241-250.

Hall, Peter A. 1989. The political power of economic ideas - Keynesianism across nations. Princeton: Princeton University Press.

Hibbs, Douglas A. 1977. Political parties and macroeconomic policy. The American Political Science Review 73:1467-1487.

Hicks, Alexander, und Duane H. Swank. 1992. Politics, Institutions, and Welfare Spending in Industrialized Democracies. American Political Science Review 86:658-674.

Howlett, Michael. 2011. Designing public policies. Principles and instruments. Oxon, New York: Routledge.

Hubo, Christiane, und Max Krott. 2010. Politiksektoren als Determinanten von Umweltkonflikten am Beispiel invasiver gebietsfremder Arten. In Umwelt- und Technikkonflikte, Hrsg. Peter H. Feindt, Thomas Saretzki, 219-238. Wiesbaden: VS.

Hubo, Christiane, und Max Krott. 2015. Macht von Politiksektoren als Chance für Wandel am Beispiel Waldnaturschutz. In Macht und Wandel in der Umweltpolitik Sonderband der Zeitschrift für Politikwissenschaft., Hrsg. Lena Partzsch, Sabine Weiland, 29-54.

Hubo, C., E. Jumpertz, M. Krott, L. Nockemann, A. Steinmann und I. Bräuer. 2007. Grundlagen für die Entwicklung einer nationalen Strategie gegen invasive gebietsfremde Arten. BfN-Skripten 213, Bonn - Bad Godesberg. www.bfn.de/fileadmin/MDB/documents/service/skript213.pdf.

Inglehart, Ronald F. 1997. Modernization and postmodernization: Cultural, economic, and political changes in 43 societies. Princeton: Princeton University Press.

Inglehart, Ronald F. 2008. Changing values among western publics, 1970-2006: post-materialist values and the shift from survival values to self-expression values. West European Politics 31:130-146.

Jann, W., und K. Wegrich. 2014. Phasenmodelle und Politikprozesse: Der Policy Cycle. In Lehrbuch der Politikfeldanalyse, 3. Aufl., Hrsg. K. Schubert, N. Bandelow, 97-132. Oldenbourg: .

Jenkins-Smith, H.C., D. Nohrstedt, C.M. Weible, und P.A. Sabatier. 2014. The advocacy coalition framework: foundations, evolution, and ongoing research. In Theories of the Policy Process, 3. Aufl., Hrsg. P.A. Sabatier, C.M. Weible, 183-223. Boulder: Westview Press.

Knill, C., und J. Tosun. 2012. Public policy. A new introduction. New York: .

Knill, C., M. Debus, und S. Heichel. 2010a. Do parties matter in internationalised policy areas? The impact of political parties on environmental policy outputs in 18 OECD countries 1970-2000. European Journal of Political Research 49:301-336.

Knill, C., K. Schulze, und J. Tosun. 2010b. Politikwandel und seine Messung in der vergleichenden Staatstätigkeitsforschung: Konzeptionelle Probleme und mögliche Alternativen. Politische Vierteljahresschrift 51:409-432.

Laver, M., und K.A. Shepsle. 1990. Coalitions and cabinet government. The American Political Science Review 84:873-890.

Leben, N. 2018. Nur mit - nicht ohne uns. Land \& Forst 171:51.

Lindblom, C. 1969. The science of „muddling through“. In Readings on modern organisations, Hrsg. A. Etzioni, 154-167. Engelwood Cliffs: Prentice Hall.

Linhard, Eric, und Johannes Raabe. 2015. Die Stärken unterschiedlicher Ministerien aus der Sicht von Politikern. Zeitschrift für Politikwissenschaft 25:159-188.

Martin, L.W., und G. Vanberg. 2004. Policing the bargain: coalition government and parliamentary scrutiny. American Journal of Political Science 48:13-27.

Meyer, P., M. Schmidt, H. Spellmann, U. Bedarff, J. Bauhus, A. Reif, und V. Späth. 2011. Aufbau eines Systems nutzungsfreier Wälder. Natur und Landschaft 86:243-249.

Müller-Brandeck-Bocquet, G. 1996. Die institutionelle Dimension der Umweltpolitik. Eine vergleichende Untersuchung zu Frankreich, Deutschland und der Europäischen Union. Baden-Baden: .

Pappi, Franz Urban, Ralf Schmitt, und Eric Linhart. 2018. Die Ministeriumsverteilung in den deutschen Landesregierungen seit dem Zweiten Weltkrieg. Zeitschrift für Parlamentsfragen 49:323-342. 
Pehle, Heinrich. 1998. Das Bundesministerium für Umwelt, Naturschutz und Reaktorsicherheit: Ausgegrenzt statt integriert? Das Institutionelle Fundament der deutschen Umweltpolitik. Wiesbaden: Deutscher Universitätsverlag.

Peters, D.M., und U. Schraml. 2014. Does background matter? Disciplinary perspectives on sustainable forest management. Biodiversity Conservation 23:3373-3389.

Rayner, J., M. Howlett, J. Wilsonc, B. Cashored, und G. Hoberge. 2001. Privileging the sub-sector: critical sub-sectors and sectoral relationships in forest policy-making. Forest Policy and Economics 2:319-332.

Sack, Detlev, und Annette E. Töller. 2018. Einleitung: Policies in den deutschen Ländern. Zeitschrift für Vergleichende Politikwissenschaft 12:603-619.

Schmidt, Manfred G., und Tobias Ostheim. 2007. Die Lehre von der Parteiendifferenz. In Der Wohlfahrtsstaat. Eine Einführung in den historischen und internationalen Vergleich, Hrsg. M.G. Schmidt, T. Ostheim, N.A. Siegel, und R. Zohlnhöfer, 51-62. Wiesbaden: VS. https://doi.org/10.1007/978-3531-90708-6.

Seeger, Bertram. 2003. Umweltpolitik in den 16 Ländern. Wahlprogramme und Regierungshandeln. Ein Bundesländervergleich. Univ., Diss. Heidelberg: .

SRU [Sachverständigenrat für Umweltfragen]. 2012. Umweltgutachten 2012, Verantwortung in einer begrenzten Welt. Berlin: SRU.

Storch, Sabine, und Georg Winkel. 2012. Waldnaturschutzpolitik und Klimawandel. In Wälder und Klimawandel: Künftige Strategien für Schutz und nachhaltige Nutzung Naturschutz und Biologische Vielfalt 125., Hrsg. M. Milad, S. Storch, H. Schaich, W. Konold, und G. Winkel, 58-71. Bonn-Bad Godesberg: Bundesamt für Naturschutz.

Töller, A.E. 2012. Warum kooperiert der Staat? Kooperative Umweltpolitik im Schatten der Hierarchie. Schriftenreihe Staatslehre und politische Verwaltung. Baden-Baden: Nomos.

Töller, A.E. 2017. Verkehrte Welt? Parteien(in)differenz in der Umweltpolitik am Beispiel der Regulierung des Frackings. Zeitschrift für Politikwissenschaft 27:131-160.

Töller, A.E. 2019. Kein Grund zum Feiern! In Zwischen Stillstand, Politikwandel und Krisenmanagement, Hrsg. R. Zohlnhöfer, T. Saalfeld, 569-590. Wiesbaden: Springer VS.

Tufte, Edward R. 1978. Political control of the economy. 2. Print. Princeton, NJ: Univ. Press.

Verbij, E. 2008. Inter-sectoral coordination in forest policy. A frame analysis of forest sectorization processes in Austria and the Netherlands. PhD thesis. Wageningen: Wageningen University.

Volkery, A. 2008. Naturschutzpolitik in den Bundesländern. In Die Politik der Bundesländer, Hrsg. A. Hildebrandt, F. Wolf, 258-273. Wiesbaden: VS.

Wenzelburger, Georg. 2015. Parteien. In Handbuch Policy-Forschung, Hrsg. Georg Wenzelburger, Reimut Zohlnhöfer, 81-112. Wiesbaden: Springer VS. https://doi.org/10.1007/978-3-658-01968-6.

Wurster, Stefan. 2010. Zukunftsvorsorge in Deutschland. Eine vergleichende Untersuchung der Bildungs-, Forschungs-, Umwelt- und Energiepolitik. Universitätsschriften Politik 171. Baden-Baden: Nomos. Zugl. Heidelberg: Univ., Diss., 2010.

Zohlnhöfer, Reimut. 2003. Institutionelle Hemmnisse für eine kohärente Wirtschaftspolitik. Aus Politik und Zeitgeschichte 18-19:9-15. 